\title{
Dependence of Temperatures and Kinetic Freeze-Out Volume on Centrality in Au-Au and Pb-Pb Collisions at High Energy
}

\author{
Muhammad Waqas $\mathbb{D}^{1}{ }^{1}$ Fu-Hu Liu $\mathbb{D}^{1},{ }^{1}$ and Zafar Wazir ${ }^{2}$ \\ ${ }^{1}$ Institute of Theoretical Physics \& State Key Laboratory of Quantum Optics and Quantum Optics Devices, Shanxi University, \\ Taiyuan, Shanxi 030006, China \\ ${ }^{2}$ Department of Physics, International Islamic University, H-10 Islamabad, Pakistan
}

Correspondence should be addressed to Fu-Hu Liu; fuhuliu@163.com

Received 12 February 2020; Revised 31 March 2020; Accepted 7 April 2020; Published 27 May 2020

Academic Editor: Antonio J. Accioly

Copyright (c) 2020 Muhammad Waqas et al. This is an open access article distributed under the Creative Commons Attribution License, which permits unrestricted use, distribution, and reproduction in any medium, provided the original work is properly cited. The publication of this article was funded by SCOAP ${ }^{3}$.

\begin{abstract}
Centrality-dependent double-differential transverse momentum spectra of negatively charged particles $\left(\pi^{-}, K^{-}\right.$, and $\left.\bar{p}\right)$ at the mid(pseudo)rapidity interval in nuclear collisions are analyzed by the standard distribution in terms of multicomponent. The experimental data measured in gold-gold ( $\mathrm{Au}-\mathrm{Au}$ ) collisions by the PHENIX Collaboration at the Relativistic Heavy Ion Collider (RHIC) and in lead-lead ( $\mathrm{Pb}-\mathrm{Pb}$ ) collisions by the ALICE Collaboration at the Large Hadron Collider (LHC) are studied. The effective temperature, initial temperature, kinetic freeze-out temperature, transverse flow velocity, and kinetic freeze-out volume are extracted from the fitting to transverse momentum spectra. We observed that the mentioned five quantities increase with the increase of event centrality due to the fact that the average transverse momentum increases with the increase of event centrality. This renders that larger momentum (energy) transfer and further multiple scattering had happened in central centrality.
\end{abstract}

\section{Introduction}

One of the most important questions in high-energy collisions is the identification of various phases of dense matter. It is expected to reach a deconfined state of matter (quarks and gluons) at high energy or density. This state of matter is called Quark-Gluon Plasma (QGP), which was obtained in the early universe shortly after the big bang prior to the condensation in hadrons. The characterization of phase transition in finite systems is a fascinating multidisciplinary topic which has been studied for decades $[1,2]$ within different phenomenological applications. The Relativistic Heavy Ion Collider (RHIC) and Large Hadron Collider (LHC) have been providing excellent tools to determine the phase structure of the strongly interacting Quantum Chromodynamics (QCD) matter [3-5] and to study the properties of QGP [6-8].

Within the framework of statistical thermal models, it is assumed that the initial stage of collisions of nuclei at the RHIC and LHC [9-11] gives a tremendous amount of temperature, where a hot and dense "fireball" over an extended region for a very short period of time (almost $10^{-22}$ seconds) is formed. The fireball consists of QGP, and it instantly cools which results in the expansion of the system (the change of the volume or density of the system), and the partons recombine into a blizzard of hadronic matter [12]. After the hadronization of the fireball, the hadrons continuously interact with each other and the particle number changes. This process results in a decrease of temperature and at a certain value where the reaction process stops and the temperature at this point is called the "chemical freeze-out temperature" $\left(T_{\mathrm{ch}}\right)$. At the stage of chemical freeze-out, the yield ratios of different types of particles remain invariant [13].

However, the rescattering process still takes place which continues to build up the collective (hydrodynamical) expansion. Resultantly, the matter becomes dilute and the mean free path of the given hadrons for the elastic reaction processes becomes comparable with the size of the system. At this stage, the rescattering process stops, which results in 
the decoupling of hadrons from the rest of the system [14]. This stage is called as the kinetic or thermal freeze-out stage, and the temperature at this stage is called the kinetic or thermal freeze-out temperature $\left(T_{0}\right)$. After this stage, the particle's energy/momentum spectrum is frozen in time and it is the least stage of the system evolution. Meanwhile, at this stage, the particle's movement is also affected by the flow effect which should be excluded when one extracts $T_{0}$. To describe the flow effect, one may use the transverse flow velocity $\beta_{\mathrm{T}}$.

The above discussed $T_{0}$ and $\beta_{\mathrm{T}}$ can be extracted from transverse momentum $\left(p_{\mathrm{T}}\right)$ spectra of particles. Also, from $p_{\mathrm{T}}$ spectra, one can extract the initial temperature $T_{i}$ according to the color string percolation model [15-17]. Generally, if the flow effect is not excluded in the temperature parameter, this type of temperature is called the effective temperature $(T)$. At least, three types of temperatures, $T, T_{i}$, and $T_{0}$, can be extracted from $p_{\mathrm{T}}$ spectra. Although the yield ratios of different types of particles can be also obtained from the normalizations of $p_{\mathrm{T}}$ spectra and then $T_{\mathrm{ch}}$ can be also extracted from $p_{\mathrm{T}}$ spectra, we mainly extract other three types of temperatures and $\beta_{\mathrm{T}}$ from $p_{\mathrm{T}}$ spectra in this paper due to their more pending situations.

In addition, volume is also an important parameter in high-energy collisions. The volume occupied by the ejectiles when the mutual interactions become negligible, and the only force they feel is the columbic repulsive force, is known as the kinetic freeze-out volume $(V)$. There are various freeze-out volumes at various freeze-out stages, but we are only focusing on the kinetic freeze-out volume $V$ in the present work. As we know, $V$ gives the information of the coexistence of phase transition and is important in the extraction of multiplicity, microcanonical heat capacity, and its negative branch or shape of the caloric curves under the thermal constraints [18-22]. In this paper, the fifth quantity extracted from $p_{\mathrm{T}}$ spectra is $V$. By way of parenthesis, the mean $p_{\mathrm{T}}$, i.e., $\left\langle p_{\mathrm{T}}\right\rangle$, is also obtained.

The study of three types of temperatures, transverse flow velocity, and kinetic freeze-out volume is a very wide, interesting, and of course huge project. However, in this paper, we will only analyze the centrality dependences of the five quantities in gold-gold (Au-Au) collisions at $200 \mathrm{GeV}$ and in lead-lead $(\mathrm{Pb}-\mathrm{Pb})$ collisions at $2.76 \mathrm{TeV}$. Only the $p_{\mathrm{T}}$ spectra of negatively charged pions $\left(\pi^{-}\right)$, kaons $\left(K^{-}\right)$, and antiprotons $(\bar{p})$ are used in the extraction. These representational spectra are enough to extract the necessary centrality dependences.

The remainder of this paper orderly consists of the method and formalism, results and discussion, and summary and conclusions which are presented in Sections 2, 3, and 4, respectively.

\section{The Method and Formalism}

Soft excitation and hard scattering processes are the two generally considered processes for the particle production. The soft excitation process contributes in a narrow $p_{\mathrm{T}}$ range which is less than $2-3 \mathrm{GeV} / c$ or up to $4-5 \mathrm{GeV} / c$ and is responsible for the production of most of the light flavored particles. The soft excitation process has various choices of formalisms including but are not limited to the Hagedorn thermal model (Statistical-Bootstrap model) [23], the standard distribution [24], the blast-wave model with BoltzmannGibbs statistics [25-27], the blast-wave model with Tsallis statistics [28-30], and the current thermodynamical-related models [31-34]. The main contributor to the produced particles is the soft excitation process.

If necessary, for the hard excitation process, there is limited choice of formalisms [35-37] and it can be described by the theory of strong interaction. In fact, the contribution of the hard scattering process is parameterized to an inverse power law, i.e., the Hagedorn function [23]:

$$
f_{\mathrm{H}}\left(p_{\mathrm{T}}\right)=A p_{\mathrm{T}}\left(1+\frac{p_{\mathrm{T}}}{p_{0}}\right)^{-n}
$$

where $p_{0}$ and $n$ are free parameters and $A$ is the normalized constant related to the free parameters. The inverse power law is obtained from the calculus of QCD [3-5] and has at least three revisions, which is out of focus of the present work and will not be discussed further.

Different probability density functions can be used to describe the contributions of the soft excitation and hard scattering processes. Due to few fractions and being earlier than the kinetic freeze-out stage, the hard scattering process does not contribute largely to $T_{0}$ and $\beta_{\mathrm{T}}$ in general. In fact, the contribution of the hard scattering process can be neglected if we study the $p_{\mathrm{T}}$ spectra in a narrow range, say $p_{\mathrm{T}}<2-3 \mathrm{GeV} / c$ or extending to $p_{\mathrm{T}}<4-5 \mathrm{GeV} / c$, for which only the contribution of the soft excitation process is indeed needed. In our opinion, various distributions show similar behaviors in case of fitting the data with acceptable representations, which results $p_{\mathrm{T}}$ in similar $\left\langle p_{\mathrm{T}}\right\rangle\left(\sqrt{\left\langle p_{\mathrm{T}}^{2}\right\rangle}\right)$ with different parameters.

For the spectra contributed by the soft excitation process, we can choose the standard distribution, as it is very close in concept to the ideal gas model. The standard distribution is the combination of the Boltzmann, Fermi-Dirac, and BoseEinstein distributions. The probability density function of the standard distribution in terms of $p_{\mathrm{T}}$ at midrapidity is generally as follows [24]:

$$
f_{S}\left(p_{\mathrm{T}}\right)=C \frac{g V^{\prime}}{(2 \pi)^{2}} p_{\mathrm{T}} \sqrt{p_{\mathrm{T}}^{2}+m_{0}^{2}} \times\left[\exp \left(\frac{\sqrt{p_{\mathrm{T}}^{2}+m_{0}^{2}}}{T}\right)+S\right]^{-1},
$$

where $C$ is the normalization constant, $V^{\prime}$ is the fitted kinetic freeze-out volume (in terms of interaction volume) of the emission source at the stage of kinetic freeze-out as discussed above, $g$ is the degeneracy factor for pion and kaon (or proton) and has the value of 3 (or 2), $m_{0}$ is the rest mass of the considered particle, $S=-1(+1)$ is for bosons (fermions), and $T$ is the effective temperature as discussed above. 
By considering the experimental rapidity range $\left[y_{\min }\right.$, $\left.y_{\max }\right]$ around the midrapidity, the accurate form of Equation (2) is as follows [24]:

$$
\begin{aligned}
f_{S}\left(p_{\mathrm{T}}\right)= & C \frac{g V^{\prime}}{(2 \pi)^{2}} p_{\mathrm{T}} \sqrt{p_{\mathrm{T}}^{2}+m_{0}^{2}} \int_{y_{\min }}^{y_{\max }} \cosh y \\
& \times\left[\exp \left(\frac{\sqrt{p_{\mathrm{T}}^{2}+m_{0}^{2}} \cosh y}{T}\right)+S\right]^{-1} d y .
\end{aligned}
$$

$T$ and $V^{\prime}$ in Equations (2) and (3) are free parameters. In most cases, the single-component standard distribution is not enough for the simultaneous description of the low- $p_{\mathrm{T}}$ region. So we have to use a two-component standard distribution.

In particular, in some cases, the multicomponent (l-component) standard distribution has to be used, which can be demonstrated as follows:

$$
\begin{aligned}
f_{S}\left(p_{\mathrm{T}}\right)= & \sum_{i=1}^{l} k_{i} C_{i} \frac{g V_{i}^{\prime}}{(2 \pi)^{2}} p_{\mathrm{T}} \sqrt{p_{\mathrm{T}}^{2}+m_{0}^{2}} \int_{y_{\min }}^{y_{\max }} \cosh y \\
& \times\left[\exp \left(\frac{\sqrt{p_{T}^{2}+m_{0}^{2}} \cosh y}{T_{i}}\right)+S\right]^{-1} d y
\end{aligned}
$$

where $C_{i}$ is the normalization constant, $k_{i}$ represents the fraction contributed by the $i$ th component, and $T_{i}$ and $V_{i}^{\prime}$ are free parameters denoted the kinetic freeze-out temperature and volume, respectively, corresponding to the $i$ th component.

In case of considering both the contributions of the soft excitation and hard scattering processes, we use the superposition in principle:

$$
f_{0}\left(p_{\mathrm{T}}\right)=k_{S} f_{S}\left(p_{\mathrm{T}}\right)+\left(1-k_{S}\right) f_{\mathrm{H}}\left(p_{\mathrm{T}}\right)
$$

where $k_{S}$ is the contribution ratio of the soft excitation process. The contribution ranges of the soft excitation and hard scattering processes described by Equation (5) are intersectant in the low- $p_{\mathrm{T}}$ region.

Another type of superposition which uses the usual step function $\theta(x)$ based on the Hagedorn model [23] is

$$
f_{0}\left(p_{T}\right)=A_{1} \theta\left(p_{1}-p_{T}\right) f_{S}\left(p_{T}\right)+A_{2} \theta\left(p_{T}-p_{1}\right) f_{\mathrm{H}}\left(p_{T}\right)
$$

where $A_{1}$ and $A_{2}$ are the normalized constants which synthesize $A_{1} f_{S}\left(p_{1}\right)=A_{2} f_{\mathrm{H}}\left(p_{1}\right)$. The contribution ranges of the soft excitation and hard scattering processes described by Equation (6) are segregative at $p_{\mathrm{T}}=p_{1}$.

In the present work, we will study only the first component in Equation (5) or (6), because we do not study a very wide $p_{\mathrm{T}}$ range. Meanwhile, we use the two-component standard distribution, i.e., $l=2$, in Equation (4) in which the first or second component has no particular priority. As probability density functions, Equations (1)-(6) are normalized to 1 naturally. When we compare the probability density functions with the experimental data which appear usually in other forms, a suitable transformation for the probability density function is certainly needed. Considering the treatment of normalization, the real fitted kinetic freeze-out volume should be $V_{i}^{\prime} /\left(C_{i} k_{i}\right)$ which will be simply used in the following section as the symbols, $V_{i}$ or $V\left(=\sum_{i=1}^{l} V_{i}\right)$.

\section{Results and Discussion}

Figure 1 presents the event centrality-dependent doubledifferential $p_{\mathrm{T}}$ spectra, $\left(1 / 2 \pi p_{\mathrm{T}}\right) d^{2} N / d p_{\mathrm{T}} d y$, of the identified particles $\left(\pi^{-}, K^{-}\right.$, and $\left.\bar{p}\right)$ produced in Au-Au collisions at $\sqrt{s_{N N}}=200 \mathrm{GeV}$ in the midpseudorapidity interval of $|\eta|$ $<0.35$, where $N$ and $y$ stands for the number of particles and rapidity, respectively. The symbols are the experimental data measured by the PHENIX Collaboration [38]. The spectra of centralities $0-5 \%, 5-10 \%, 10-15 \%, 15-20 \%, 20-30 \%$, $30-40 \%, 40-50 \%, 50-60 \%, 60-70 \%, 70-80 \%$, and $80-92 \%$ in the three panels are multiplied by $20,10,5,2.5,1.5,1,1,1$, 1,1 , and 1 , respectively. In addition, the spectra of centralities $15-20 \%$, 20-30\%, 30-40\%, 40-50\%, 50-60\%, 60-70\%, $70-80 \%$, and $80-92 \%$ in panel (a) are remultiplied by 0.8 , $0.7,0.6,0.45,0.3,0.24,0.17$, and 0.15 , respectively. The curves are our fitting results by using the two-component standard distribution, Equation (4) with $l=2$. The substantially togethered event centralities, the values of free parameters $\left(T_{1}, T_{2}, V_{1}\right.$, and $\left.V_{2}\right), \chi^{2}$, and number of degree of freedom (ndof) are listed in Table 1. One can see the wellapproximate description of the model results to the experimental data of the PHENIX Collaboration in special $p_{\mathrm{T}}$ ranges in high-energy $\mathrm{Au}-\mathrm{Au}$ collisions at the RHIC.

Figure 2 is the same as Figure 1, but it gives the results for $\pi^{-}, K$, and $\bar{p}$ in different centrality bins in $\mathrm{Pb}-\mathrm{Pb}$ collisions at $2.76 \mathrm{TeV}$ in the midrapidity interval $|y|<0.5$. The experimental data of the ALICE Collaboration is represented by the symbols [39], whereas the spectra of $\mathrm{Pb}-\mathrm{Pb}$ are scaled by the factor of $2^{n}$ and $n$ changes from 9 to 0 with the change of event centrality such as from $0-5 \%$ to $80-90 \%$. The related parameters and the existing centralities are listed together in Table 1. One can see the well-approximate description of the model results to the experimental data of the ALICE Collaboration in special $p_{\mathrm{T}}$ ranges in high-energy $\mathrm{Pb}-\mathrm{Pb}$ collisions at the LHC.

To study the change trend of parameters with centrality, Figure 3 shows the dependence of effective temperature on centrality for the productions of $\pi^{-}, K^{-}$, and $\bar{p}$ in different centrality bins in the $\mathrm{Au}-\mathrm{Au}$ and $\mathrm{Pb}-\mathrm{Pb}$ collisions at $200 \mathrm{GeV}$ and $2.76 \mathrm{TeV}$, respectively. Panel (a) shows the result for Au-Au collisions, while panel (b) shows the result for $\mathrm{Pb}-\mathrm{Pb}$ collisions. Different symbols represent different particles. One can see the clear decrease of effective temperature from the central to peripheral collisions. The reason behind it is as follows: the more violent collisions in central collisions where it can get a degree of higher excitation and also involve more number of participants in interactions, while they decrease from the central to peripheral collisions. The effective temperatures in collisions at the LHC in different centrality bins are higher than those at the RHIC due to more energy deposition in collisions at the LHC. 


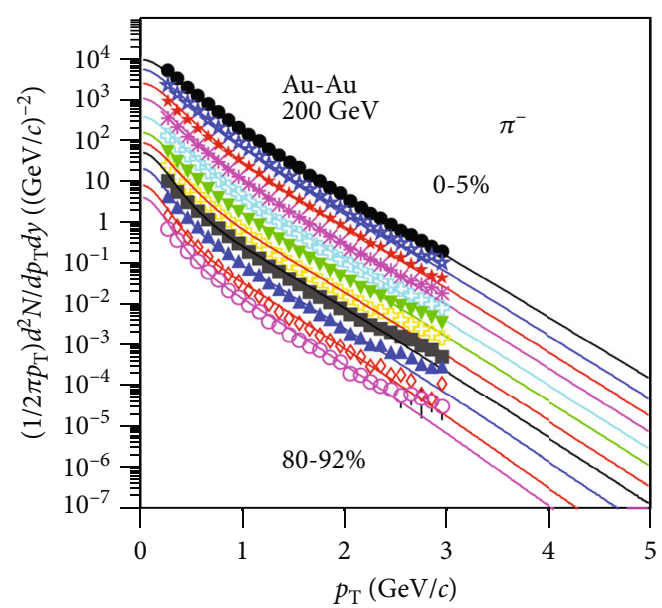

(a)

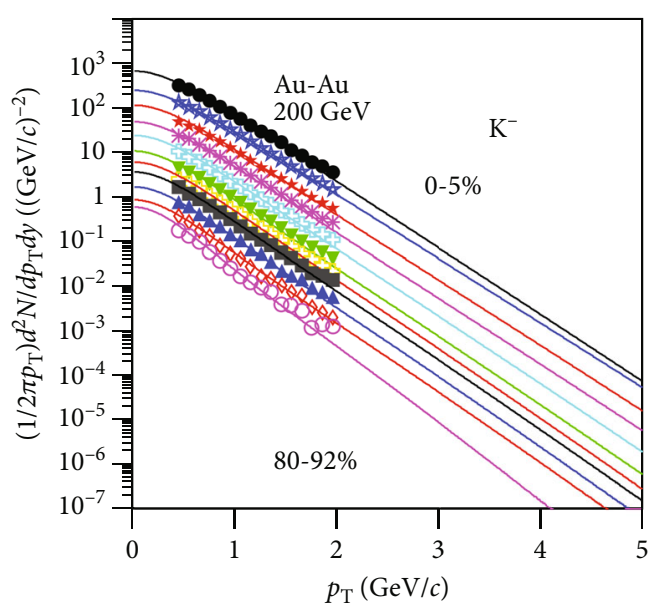

(b)

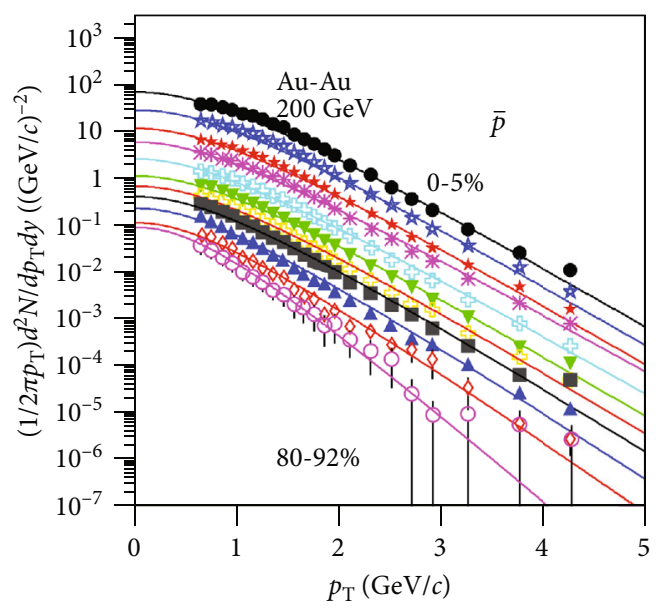

(c)

Figure 1: Centrality-dependent $\left(1 / 2 \pi p_{\mathrm{T}}\right) d^{2} N / d p_{\mathrm{T}} d y$ of (a) $\pi^{-}$, (b) $K^{-}$, and (c) $\bar{p}$ produced in $|\eta|<0.35$ in Au-Au collisions at $200 \mathrm{GeV}$. The symbols represent experimental data of the PHENIX Collaboration [38], while the curves are the results of our fits by using the twocomponent standard distribution, Equation (4) with $l=2$. The spectra of centralities $0-5 \%, 5-10 \%, 10-15 \%, 15-20 \%, 20-30 \%, 30-40 \%$, $40-50 \%, 50-60 \%, 60-70 \%, 70-80 \%$, and $80-92 \%$ in the three panels are multiplied by $20,10,5,2.5,1.5,1,1,1,1,1$, and 1 , respectively. In addition, the spectra of centralities $15-20 \%, 20-30 \%, 30-40 \%, 40-50 \%, 50-60 \%, 60-70 \%, 70-80 \%$, and $80-92 \%$ in panel (a) are remultiplied by $0.8,0.7,0.6,0.45,0.3,0.24,0.17$, and 0.15 , respectively.

Figure 4 is the same as Figure 3; however, it shows the result for the dependence of kinetic freeze-out volume $V$ in events with different centralities $C$, where $V=V_{1}+V_{2}$ due to the additivity of volume. One can see that the kinetic freeze-out volume decreases from the central to peripheral collisions, as the number of participant nucleons decreases from the central to peripheral collisions depending on the interaction volume. Due to the large number of binary collisions by the rescattering of partons, the system with more participants reaches quickly to an equilibrium state, but because of the decreases in centrality and the decreases in the number of participants, the system goes slowly to an equilibrium state. The large volume and more number of participants in the central collisions may indicate the occupation of superdense hadronic matter, but of course, further and more complete information about the local energy density of superhadronic matter is needed to study the possible phase transition of QGP. The figure shows a volume differen- tial scenario too. The heavier the particle is, the smaller the kinetic freeze-out volume has, which shows the early freezeout of heavier particles as compared to the lighter particles and suggests different freeze-out surfaces for different particles. Such result can be found in the literature $[40,41]$.

The dependence of the mean transverse momentum $\left\langle p_{\mathrm{T}}\right\rangle$ in different centrality events is shown in Figure 5. The symbols represent $\left\langle p_{\mathrm{T}}\right\rangle$ for different particles obtained from the fitting function Equation (4) with $l=2$ over a $p_{\mathrm{T}}$ range from 0 to $5 \mathrm{GeV} / c$, where the parameter values are listed in Table 1 . One can see that $\left\langle p_{\mathrm{T}}\right\rangle$ decreases from the central to peripheral $\mathrm{Au}-\mathrm{Au}$ and $\mathrm{Pb}-\mathrm{Pb}$ collisions for all particle species, and it may be caused due to decreasing the participant nucleons from the central to peripheral collisions and this result is similar to Ref. [42]. It is also important to notice that $\bar{p}$ spectra exhibit a concave shape in the peripheral events, which is well described by the power-law parametrization as observed in Ref. [43], but this curvature decreases with the increasing 
TABLE 1: Values of effective temperatures $\left(T_{1}\right.$ and $\left.T_{2}\right)$, volumes $\left(V_{1}\right.$ and $\left.V_{2}\right), \chi^{2}$, and number of degree of freedom (ndof) corresponding to the curves in Figures 1 and 2.

\begin{tabular}{|c|c|c|c|c|c|c|c|c|}
\hline Figure & Particle & Centrality & $T_{1}(\mathrm{GeV})$ & $T_{2}(\mathrm{GeV})$ & $V_{1}\left((\mathrm{fm})^{3}\right)$ & $V_{2}\left((\mathrm{fm})^{3}\right)$ & $\chi^{2}$ & ndof \\
\hline \multirow{33}{*}{$\begin{array}{l}\text { Figure } 1 \\
\mathrm{Au}-\mathrm{Au} \\
200 \mathrm{GeV}\end{array}$} & \multirow{11}{*}{$\pi^{-}$} & $0-5 \%$ & $0.181 \pm 0.014$ & $0.268 \pm 0.006$ & $745 \pm 100$ & $1967 \pm 309$ & 45 & 24 \\
\hline & & $5-10 \%$ & $0.138 \pm 0.013$ & $0.270 \pm 0.006$ & $564 \pm 80$ & $2054 \pm 174$ & 14 & 24 \\
\hline & & $10-15 \%$ & $0.179 \pm 0.015$ & $0.250 \pm 0.005$ & $430 \pm 62$ & $2004 \pm 153$ & 233 & 24 \\
\hline & & $15-20 \%$ & $0.167 \pm 0.012$ & $0.247 \pm 0.003$ & $354 \pm 61$ & $1862 \pm 320$ & 63 & 24 \\
\hline & & $20-30 \%$ & $0.151 \pm 0.009$ & $0.245 \pm 0.004$ & $360 \pm 39$ & $1854 \pm 216$ & 87 & 24 \\
\hline & & $30-40 \%$ & $0.125 \pm 0.015$ & $0.237 \pm 0.006$ & $195 \pm 30$ & $2005 \pm 189$ & 276 & 24 \\
\hline & & $40-50 \%$ & $0.148 \pm 0.014$ & $0.227 \pm 0.004$ & $106 \pm 25$ & $1585 \pm 171$ & 388 & 24 \\
\hline & & $50-60 \%$ & $0.098 \pm 0.011$ & $0.227 \pm 0.003$ & $59 \pm 11$ & $1048 \pm 153$ & 146 & 24 \\
\hline & & $60-70 \%$ & $0.125 \pm 0.010$ & $0.274 \pm 0.006$ & $37 \pm 11$ & $984 \pm 109$ & 71 & 24 \\
\hline & & $70-80 \%$ & $0.112 \pm 0.014$ & $0.209 \pm 0.007$ & $18 \pm 3$ & $296 \pm 37$ & 256 & 24 \\
\hline & & $80-92 \%$ & $0.068 \pm 0.015$ & $0.221 \pm 0.006$ & $8.0 \pm 1.0$ & $213 \pm 13$ & 69 & 24 \\
\hline & \multirow{11}{*}{$K^{-}$} & $0-5 \%$ & $0.201 \pm 0.009$ & $0.271 \pm 0.008$ & $196 \pm 36$ & $1081 \pm 178$ & 17 & 12 \\
\hline & & $5-10 \%$ & $0.188 \pm 0.007$ & $0.255 \pm 0.008$ & $32 \pm 6$ & $1116 \pm 71$ & 18 & 12 \\
\hline & & $10-15 \%$ & $0.211 \pm 0.013$ & $0.250 \pm 0.006$ & $37 \pm 6$ & $1030 \pm 102$ & 24 & 12 \\
\hline & & $15-20 \%$ & $0.135 \pm 0.014$ & $0.248 \pm 0.008$ & $35 \pm 5$ & $836 \pm 105$ & 14 & 12 \\
\hline & & $20-30 \%$ & $0.219 \pm 0.015$ & $0.241 \pm 0.006$ & $10 \pm 1$ & $733 \pm 66$ & 18 & 12 \\
\hline & & $30-40 \%$ & $0.156 \pm 0.010$ & $0.238 \pm 0.007$ & $4.0 \pm 0.3$ & $665 \pm 82$ & 13 & 12 \\
\hline & & $40-50 \%$ & $0.130 \pm 0.011$ & $0.235 \pm 0.008$ & $13 \pm 1.2$ & $253 \pm 39$ & 24 & 12 \\
\hline & & $50-60 \%$ & $0.170 \pm 0.013$ & $0.230 \pm 0.006$ & $1.6 \pm 0.2$ & $577 \pm 48$ & 9 & 12 \\
\hline & & $60-70 \%$ & $0.163 \pm 0.015$ & $0.225 \pm 0.006$ & $0.50 \pm 0.02$ & $544 \pm 50$ & 35 & 12 \\
\hline & & $70-80 \%$ & $0.155 \pm 0.015$ & $0.220 \pm 0.007$ & $0.30 \pm 0.01$ & $505 \pm 60$ & 215 & 12 \\
\hline & & $80-92 \%$ & $0.130 \pm 0.010$ & $0.218 \pm 0.005$ & $0.030 \pm 0.001$ & $460 \pm 130$ & 66 & 12 \\
\hline & \multirow{11}{*}{$\bar{p}$} & $0-5 \%$ & $0.280 \pm 0.017$ & $0.320 \pm 0.003$ & $36 \pm 7$ & $786 \pm 120$ & 17 & 18 \\
\hline & & $5-10 \%$ & $0.272 \pm 0.015$ & $0.317 \pm 0.002$ & $31 \pm 7$ & $775 \pm 70$ & 20 & 18 \\
\hline & & $10-15 \%$ & $0.265 \pm 0.018$ & $0.313 \pm 0.006$ & $16 \pm 3$ & $747 \pm 86$ & 162 & 18 \\
\hline & & $15-20 \%$ & $0.185 \pm 0.016$ & $0.313 \pm 0.005$ & $23 \pm 4$ & $710 \pm 80$ & 35 & 18 \\
\hline & & $20-30 \%$ & $0.211 \pm 0.013$ & $0.309 \pm 0.006$ & $13 \pm 1$ & $678 \pm 6541$ & 41 & 18 \\
\hline & & $30-40 \%$ & $0.247 \pm 0.018$ & $0.317 \pm 0.007$ & $10 \pm 1$ & $634 \pm 50$ & 11 & 18 \\
\hline & & $40-50 \%$ & $0.244 \pm 0.015$ & $0.308 \pm 0.008$ & $4.0 \pm 0.5$ & $597 \pm 37$ & 39 & 18 \\
\hline & & $50-60 \%$ & $0.237 \pm 0.013$ & $0.300 \pm 0.004$ & $1.6 \pm 0.3$ & $574 \pm 70$ & 43 & 18 \\
\hline & & $60-70 \%$ & $0.210 \pm 0.013$ & $0.285 \pm 0.006$ & $0.50 \pm 0.02$ & $476 \pm 45$ & 28 & 18 \\
\hline & & $70-80 \%$ & $0.170 \pm 0.014$ & $0.267 \pm 0.005$ & $0.30 \pm 0.01$ & $455 \pm 50$ & 110 & 18 \\
\hline & & $80-92 \%$ & $0.172 \pm 0.010$ & $0.218 \pm 0.004$ & $0.40 \pm 0.01$ & $406 \pm 40$ & 5 & 18 \\
\hline \multirow{9}{*}{$\begin{array}{l}\text { Figure } 2 \\
\mathrm{~Pb}-\mathrm{Pb} \\
2.76 \mathrm{TeV}\end{array}$} & \multirow{9}{*}{$\pi^{-}$} & $0-5 \%$ & $0.179 \pm 0.010$ & $0.340 \pm 0.004$ & $2517 \pm 208$ & $4099 \pm 252$ & 206 & 37 \\
\hline & & $5-10 \%$ & $0.172 \pm 0.012$ & $0.331 \pm 0.004$ & $2226 \pm 135$ & $3468 \pm 201$ & 101 & 37 \\
\hline & & $10-20 \%$ & $0.165 \pm 0.010$ & $0.312 \pm 0.005$ & $1395 \pm 70$ & $2813 \pm 176$ & 70 & 37 \\
\hline & & $20-30 \%$ & $0.161 \pm 0.014$ & $0.300 \pm 0.006$ & $1012 \pm 103$ & $2427 \pm 231$ & 94 & 37 \\
\hline & & $30-40 \%$ & $0.147 \pm 0.013$ & $0.280 \pm 0.005$ & $534 \pm 56$ & $3502 \pm 165$ & 244 & 37 \\
\hline & & $40-50 \%$ & $0.129 \pm 0.012$ & $0.289 \pm 0.005$ & $307 \pm 38$ & $3600 \pm 198$ & 110 & 37 \\
\hline & & $50-60 \%$ & $0.108 \pm 0.010$ & $0.281 \pm 0.005$ & $176 \pm 32$ & $3380 \pm 213$ & 206 & 37 \\
\hline & & $60-70 \%$ & $0.114 \pm 0.015$ & $0: 290 \pm 0: 005$ & $94 \pm 13$ & $3150 \pm 147$ & 125 & 35 \\
\hline & & $70-80 \%$ & $0.130 \pm 0.010$ & $0.287 \pm 0.007$ & $45 \pm 9$ & $2930 \pm 206$ & 502 & 35 \\
\hline
\end{tabular}


TABLE 1: Continued.

\begin{tabular}{|c|c|c|c|c|c|c|c|c|}
\hline Figure & Particle & Centrality & $T_{1}(\mathrm{GeV})$ & $T_{2}(\mathrm{GeV})$ & $V_{1}\left((\mathrm{fm})^{3}\right)$ & $V_{2}\left((\mathrm{fm})^{3}\right)$ & $\chi^{2}$ & ndof \\
\hline & \multirow{11}{*}{$K^{-}$} & $80-90 \%$ & $0.118 \pm 0.014$ & $0.287 \pm 0.006$ & $16 \pm 2$ & $1945 \pm 102$ & 119 & 35 \\
\hline & & $0-5 \%$ & $0.277 \pm 0.010$ & $0.361 \pm 0.007$ & $170 \pm 36$ & $3442 \pm 208$ & 3 & 32 \\
\hline & & $5-10 \%$ & $0.270 \pm 0.009$ & $0.359 \pm 0.009$ & $152 \pm 10$ & $3237 \pm 240$ & 15 & 32 \\
\hline & & $10-20 \%$ & $0.252 \pm 0.009$ & $0.354 \pm 0.007$ & $97 \pm 11$ & $3190 \pm 187$ & 8 & 32 \\
\hline & & $20-30 \%$ & $0.265 \pm 0.009$ & $0.350 \pm 0.006$ & $53 \pm 7$ & $3136 \pm 200$ & 22 & 32 \\
\hline & & $30-40 \%$ & $0.247 \pm 0.008$ & $0.395 \pm 0.006$ & $27 \pm 4$ & $3014 \pm 198$ & 12 & 32 \\
\hline & & $40-50 \%$ & $0.210 \pm 0.008$ & $0.341 \pm 0.006$ & $22 \pm 4$ & $2828 \pm 190$ & 119 & 32 \\
\hline & & $50-60 \%$ & $0.213 \pm 0.011$ & $0.345 \pm 0.005$ & $8.1 \pm 1.0$ & $2659 \pm 210$ & 26 & 32 \\
\hline & & $60-70 \%$ & $0.226 \pm 0.009$ & $0.335 \pm 0.005$ & $5.8 \pm 1.3$ & $2132 \pm 176$ & 46 & 32 \\
\hline & & $70-80 \%$ & $0.225 \pm 0.010$ & $0.332 \pm 0.006$ & $2.6 \pm 0.3$ & $1736 \pm 134$ & 71 & 32 \\
\hline & & $80-90 \%$ & $0.170 \pm 0.009$ & $0.320 \pm 0.006$ & $0.50 \pm 0.05$ & $1150 \pm 120$ & 75 & 32 \\
\hline & \multirow{10}{*}{$\bar{p}$} & $0-5 \%$ & $0.426 \pm 0.012$ & $0.421 \pm 0.007$ & $55 \pm 6$ & $1965 \pm 195$ & 49 & 33 \\
\hline & & $5-10 \%$ & $0.300 \pm 0.010$ & $0.425 \pm 0.005$ & $75 \pm 6$ & $1782 \pm 164$ & 373 & 33 \\
\hline & & $10-20 \%$ & $0.427 \pm 0.010$ & $0.397 \pm 0.352$ & $50 \pm 5$ & $1748 \pm 140$ & 43 & 33 \\
\hline & & $20-30 \%$ & $0.405 \pm 0.013$ & $0.395 \pm 0.006$ & $31 \pm 4$ & $1690 \pm 130$ & 52 & 33 \\
\hline & & $30-40 \%$ & $0.400 \pm 0.012$ & $0.382 \pm 0.005$ & $29 \pm 4$ & $1534 \pm 158$ & 20 & 33 \\
\hline & & $40-50 \%$ & $0.352 \pm 0.011$ & $0.383 \pm 0.006$ & $15 \pm 2$ & $401 \pm 135$ & 109 & 33 \\
\hline & & $50-60 \%$ & $0.331 \pm 0.012$ & $0.380 \pm 0.005$ & $6.0 \pm 0.5$ & $1357 \pm 125$ & 67 & 33 \\
\hline & & $60-70 \%$ & $0.310 \pm 0.012$ & $0.369 \pm 0.006$ & $8.7 \pm 1.0$ & $1069 \pm 90$ & 75 & 33 \\
\hline & & $70-80 \%$ & $0.321 \pm 0.015$ & $0.333 \pm 0.004$ & $1.0 \pm 0.3$ & $978 \pm 50$ & 104 & 33 \\
\hline & & $80-90 \%$ & $0.310 \pm 0.014$ & $0.328 \pm 0.006$ & $0.70 \pm 0.04$ & $708 \pm 60$ & 122 & 33 \\
\hline
\end{tabular}

centrality and it leads to an almost exponential dependence on $\left\langle p_{\mathrm{T}}\right\rangle$ for the most central collisions. Furthermore, $\left\langle p_{\mathrm{T}}\right\rangle$ for heavier particles is larger than that for lighter ones, and $\left\langle p_{\mathrm{T}}\right\rangle$ at LHC energy is slightly larger than that at RHIC energy. The increase of $\left\langle p_{\mathrm{T}}\right\rangle$ in central collisions and with the massive mass of the particle may indicate the collective radial flow, and the same behavior is observed at a few $\mathrm{GeV}$ [44] and more than $10 \mathrm{GeV}$ [45].

Figure 6 is the same as Figure 5; however, it demonstrates the result for the initial temperature $T_{i}$, where $T_{i}$ is obtained by the root-mean-square $p_{\mathrm{T}}$ divided by $\sqrt{2}$, i.e., $\sqrt{\left\langle p_{\mathrm{T}}^{2}\right\rangle / 2}$, according to the color string percolation model [15-17]. The symbols are the representation of the results obtained from the fitting function Equation (4) with $l=2$ over a $p_{\mathrm{T}}$ range in $0-5 \mathrm{GeV} / c$ and with the parameter values listed in Table 1. The mass differential temperature scenario is also observed. It is necessary to point out that the initial temperature obtained in this work is larger than the effective temperature which is in agreement with the order of time evolution of the interacting system.

Figure 7 shows the dependence of $(a, b)$ effective temperature $T$ on the rest mass $m_{0}$ and (c, d) mean transverse momentum $\left\langle p_{T}\right\rangle$ on the mean energy or mean moving mass $\bar{m}$ in the rest frame of the emission source for (a, c) Au-Au collisions at $200 \mathrm{GeV}$ and (b, d) $\mathrm{Pb}-\mathrm{Pb}$ collisions at $2.76 \mathrm{TeV}$ in different centrality classes. The symbols represent the derived quantities according to the free parameters listed in Table 1, where $T=\left(T_{1} V_{1}+T_{2} V_{2}\right) /\left(V_{1}+V_{2}\right)$, $\left\langle p_{\mathrm{T}}\right\rangle=\int p_{\mathrm{T}} f_{S}\left(p_{\mathrm{T}}\right) d p_{\mathrm{T}}$, and $\bar{m}=\left\langle\sqrt{p_{\mathrm{T}}^{2}+p_{z}^{2}+m_{0}^{2}}\right\rangle$, where $p_{z}$ is the longitudinal momentum and its distribution can be obtained according to $p_{\mathrm{T}}$ distribution if we assume isotropic emission in the source rest frame [46]. The lines are the results fitted for the values of derived quantities if we assume linear correlations are existent.

The intercepts in Figures 7(a) and 7(b) are regarded as the kinetic freeze-out temperature $T_{0}$, and the slopes in Figures $7(\mathrm{c})$ and $7(\mathrm{~d})$ are regarded as the transverse flow velocity $\beta_{\mathrm{T}}$ [47-50]. The dependences of (a) $T_{0}$ on $C$ and (b) $\beta_{\mathrm{T}}$ on $C$, as well as the correlation between (c) $\beta_{\mathrm{T}}$ and $\beta_{\mathrm{T}}$, are presented in Figure 8 . One can see that $T_{0}$ and $\beta_{\mathrm{T}}$ decrease with the increase of $C$, and $T_{0}$ increases with the increase of $\beta_{\mathrm{T}}$, which renders that central collisions display higher excitation and quicker expansion than peripheral collisions due to more energy deposition in central collisions.

It should be noted that $T_{0}$ shown in Figure 8 is larger than $160-170 \mathrm{MeV}$ which is regarded as the chemical freeze-out temperature $T_{\mathrm{ch}}$ of phase transition from hadronic matter to QGP. As expected, $T_{0}$ should be less than or equal to $T_{\mathrm{ch}}$ due to time evolution. The difference between our results and $T_{\mathrm{ch}}$ is regarded as different "thermometers" (methods) used in the extraction of temperature. In our opinion, a unified "thermometer" (method) should be used in the extraction of temperature. Or, one may find a relation to 


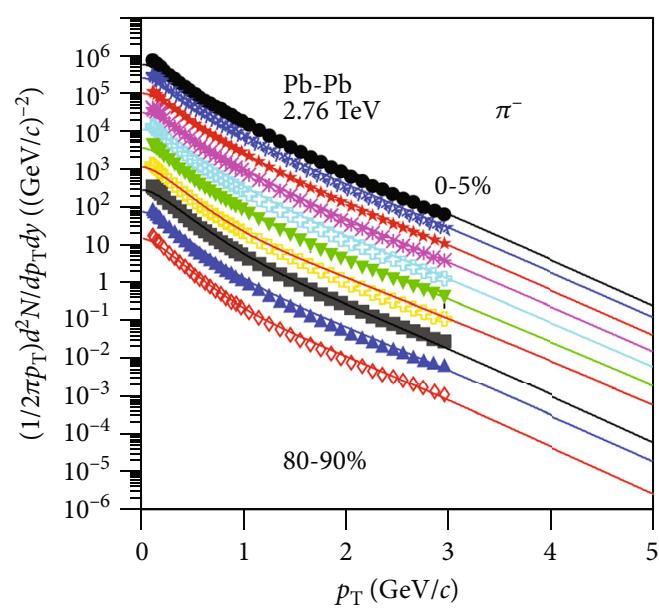

(a)

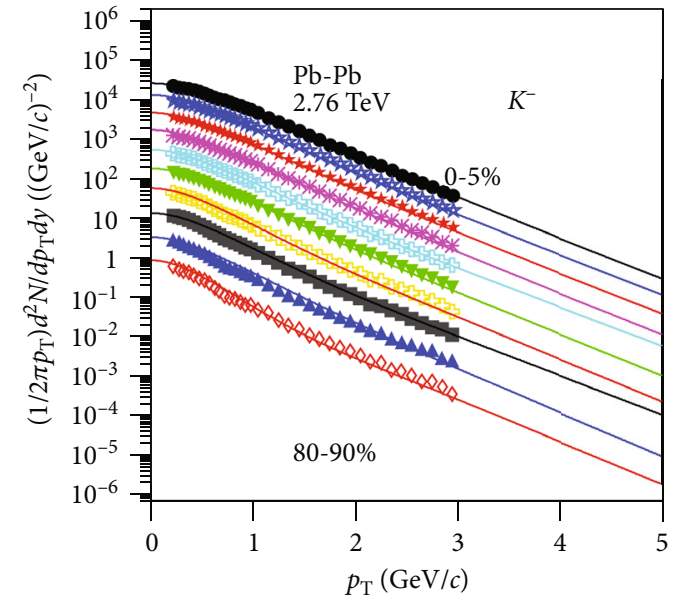

(b)

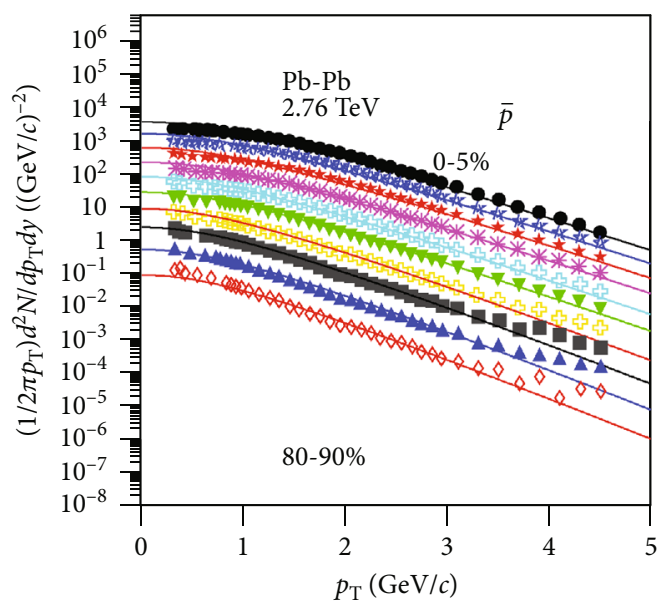

(c)

Figure 2: Same as Figure 1 but for the spectra of $\pi^{-}, K^{-}$, and $\bar{p}$ in $|y|<0.5$ in $\mathrm{Pb}-\mathrm{Pb}$ collisions at $\sqrt{s_{N N}}=2.76 \mathrm{TeV}$. The symbols represent the measured data of the ALICE Collaboration [39], where the spectra are scaled by factors $2^{n}$ and $n$ changes from 9 to 0 as the event centrality changes from $0-5 \%$ to $80-90 \%$.

convert one temperature to another one, as what one did between Celsius temperature and degree Fahrenheit.

Meanwhile, there is a positive correlation between $T_{0}$ and $\beta_{\mathrm{T}}$ as shown in Figure 8(c). Some studies show a negative correlation between $T_{0}$ and $\beta_{\mathrm{T}}$ when one uses the blastwave model [25-30]. At present, one could not decide which correlation is correct. In our opinion, for a given $p_{\mathrm{T}}$ spectrum, $T_{0}$ and $\beta_{\mathrm{T}}$ are negatively correlative if one uses the blast-wave model which gives a large $T_{0}$ to correspond to a small $\beta_{\mathrm{T}}$. However, for a set of $p_{\mathrm{T}}$ spectra with varying centralities and energies, the situation is dissimilar. The present work uses an alternative method to extract $T_{0}$ and $\beta_{\mathrm{T}}$ and obtain a positive correlation.

In addition, one can see some fluctuations in Figures 3-6 and 8 . These fluctuations have no particular physics meaning but reflect the statistical and/or systematical fluctuations in the data itself. Although there are fluctuations in the dependences of parameters on centrality, one can see the general decreasing trend of parameters when decreasing the centrality. The considered parameters have a similar trend due to their consistent meaning on the energy deposition which is reflected in terms of the excitation and expansion degree.

Both the mean transverse momentum $\left\langle p_{\mathrm{T}}\right\rangle$ and the initial temperature $T_{i}$ are obtained from $p_{\mathrm{T}}$ spectra. The relation of $\left\langle p_{\mathrm{T}}\right\rangle$ and $T_{i}$ is certainly positive correlation. Figure 9 shows this correlation. One can see that $\left\langle p_{\mathrm{T}}\right\rangle$ increases with the increase of $T_{i}$. This correlation is natural since $T_{i}$ is defined by $\sqrt{\left\langle p_{\mathrm{T}}^{2}\right\rangle / 2}$. One can also see that $\sqrt{\left\langle p_{\mathrm{T}}^{2}\right\rangle / 2}$ increases with the increase of collision energy and the size of the system, but the latter has a very small effect, so we can neglect it.

From Figures 4-9, one can see that the considered quantities in the most peripheral $\mathrm{Pb}-\mathrm{Pb}$ collisions at $2.76 \mathrm{TeV}$ overlap the most central Au-Au collisions with lower energy of $200 \mathrm{GeV}$, which may be the indication of the formation of similar systems in the most peripheral collisions at higher energies and in the most central collisions at lower energies, and it may support the hypothesis of the effective energy for the particle production [51-54].

In the considered $\mathrm{Au}-\mathrm{Au}$ collisions at $200 \mathrm{GeV}$ and $\mathrm{Pb}-\mathrm{Pb}$ collisions at $2.76 \mathrm{TeV}$, the decreasing trend of temperatures 


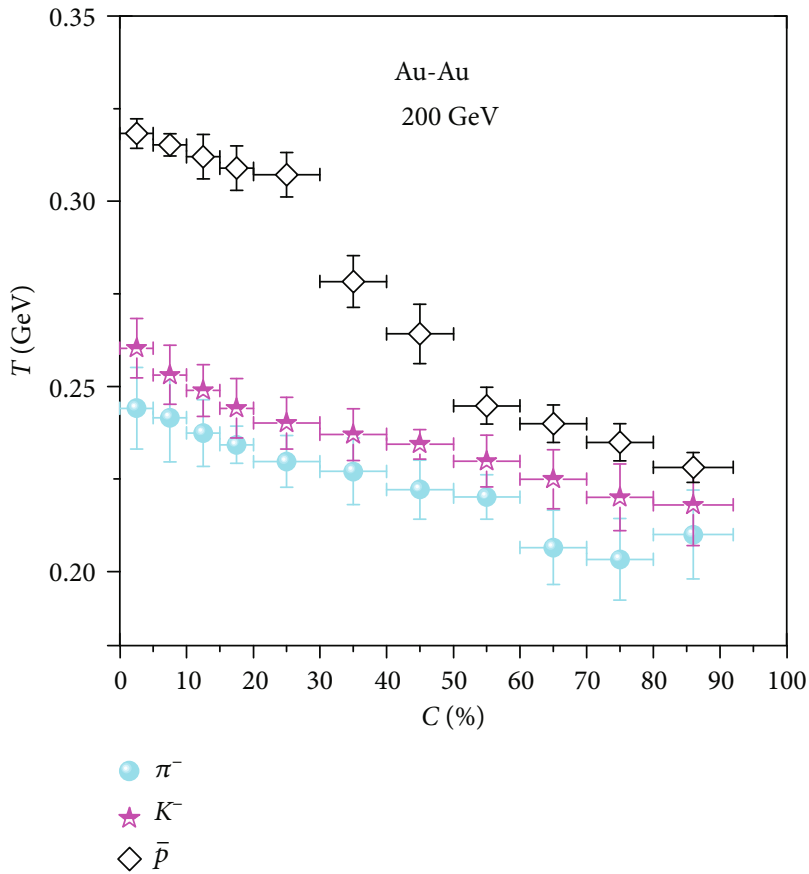

(a)

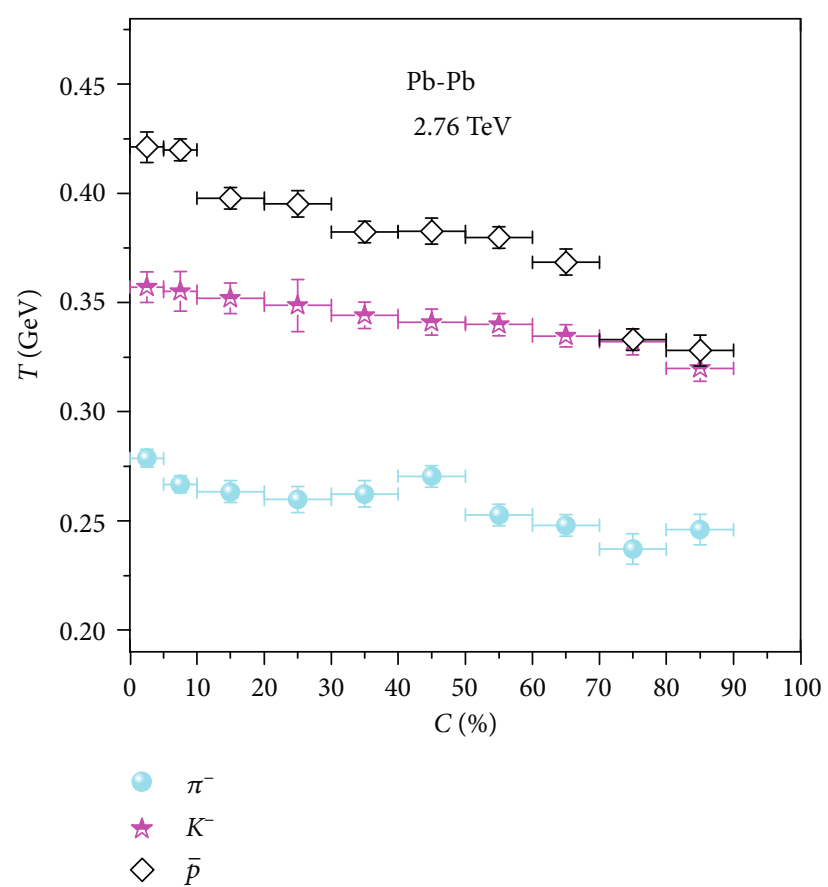

(b)

Figure 3: Dependence of effective temperature $T$ in different centrality bins $C$ in (a) Au-Au collisions at $200 \mathrm{GeV}$ and (b) $\mathrm{Pb}$ - $\mathrm{Pb}$ collisions at $2.76 \mathrm{TeV}$. The obtained values corresponded to the identified particles extracted from the experimental $p_{\mathrm{T}}$ spectra.

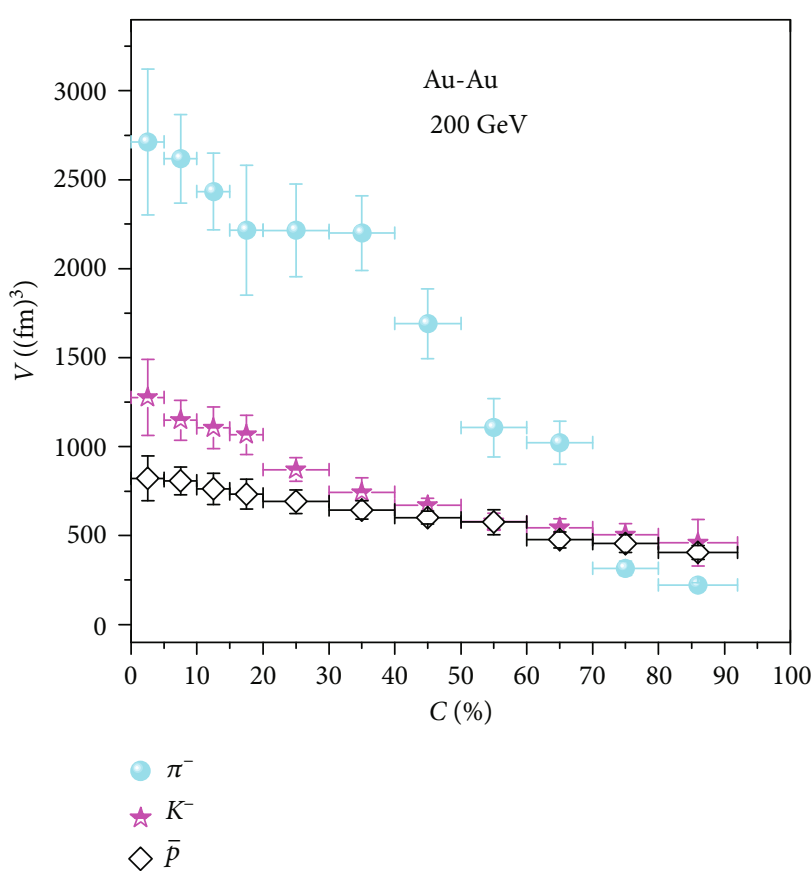

(a)

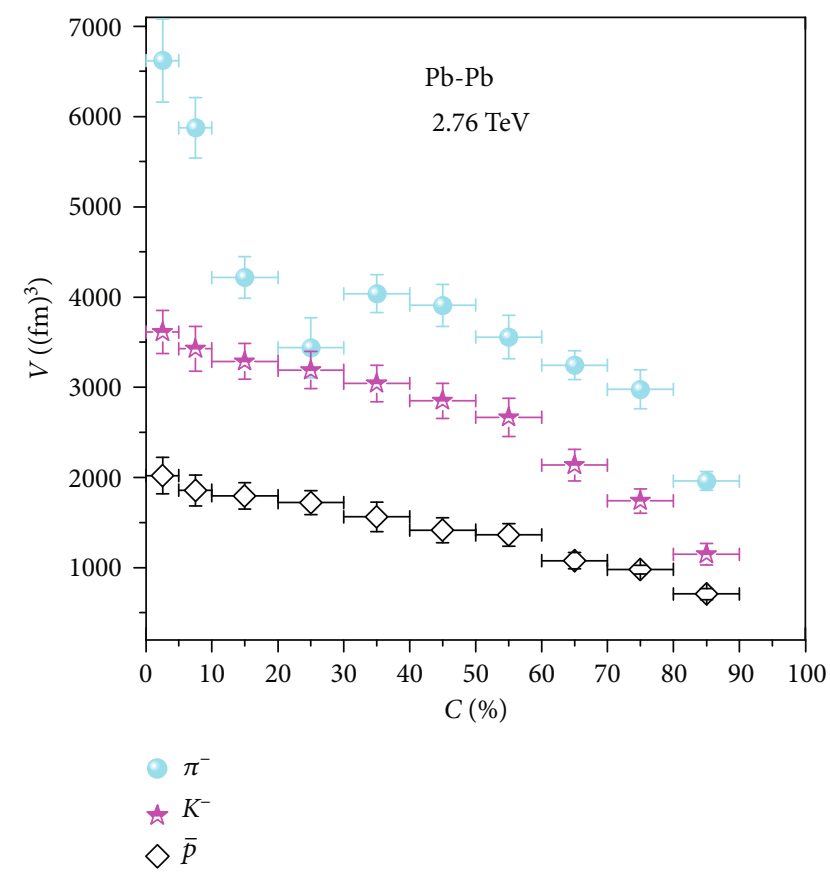

(b)

Figure 4: Same as Figure 3, but for dependence of kinetic freeze-out volume $V$ on centrality $C$.

and kinetic freeze-out volume from the central to peripheral collisions renders that more energy deposition and then higher excitation and quicker expansion in central collisions. Some studies $[9,13,55-57]$ which use other methods such as the blast-wave model show that the kinetic freeze-out temperature in central collisions is less than that in peripheral collisions, though this opposite result can be explained as longer freeze-out time in central collisions. 


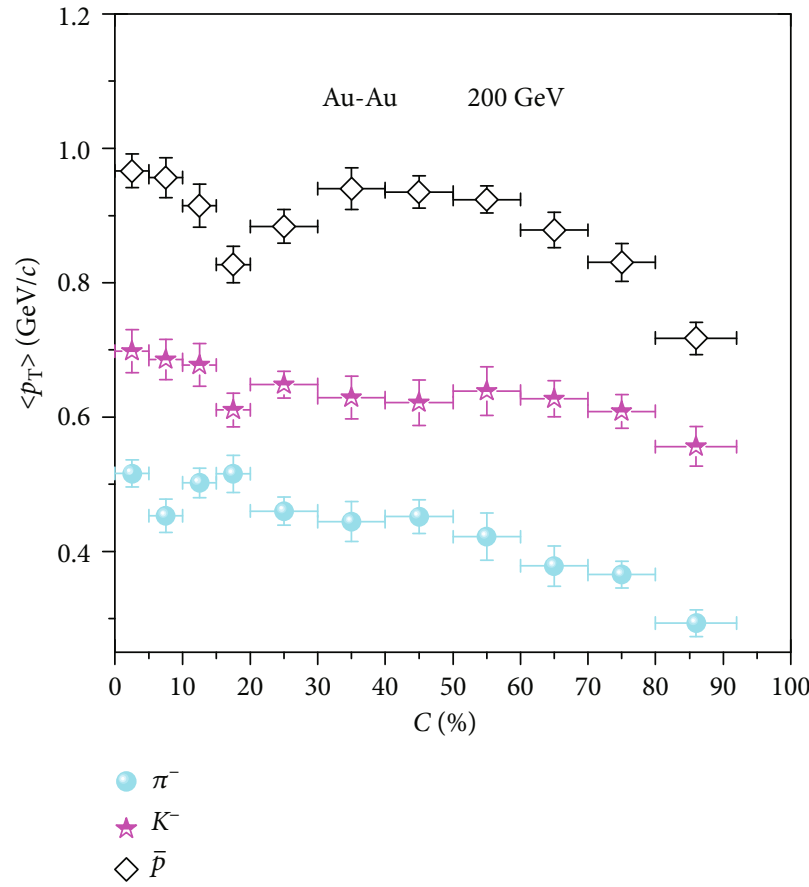

(a)

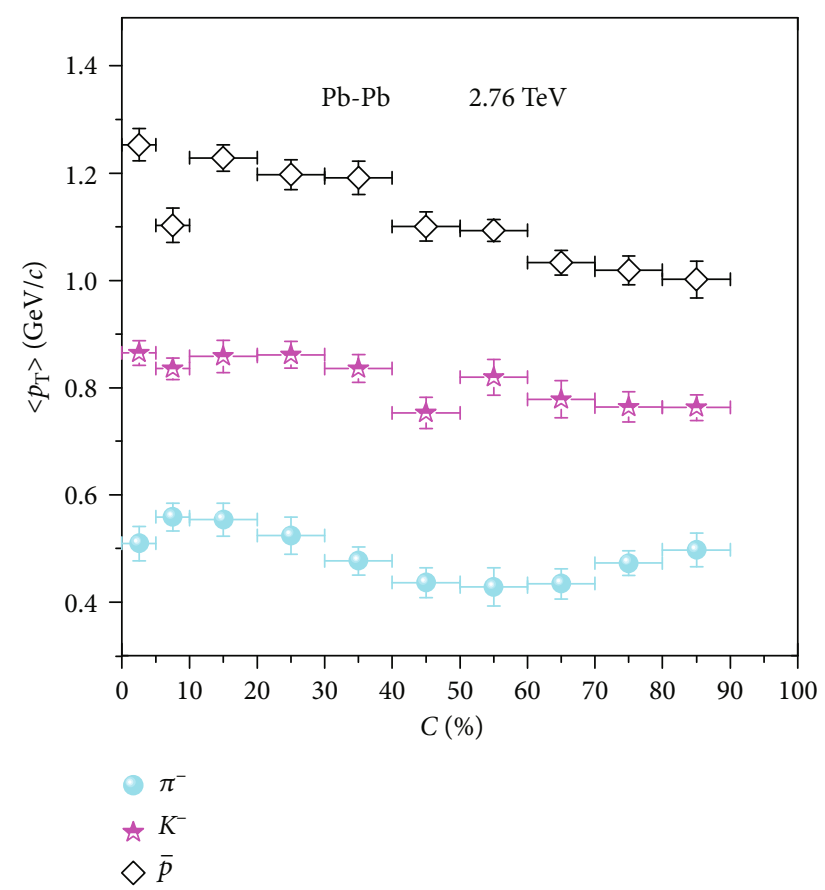

(b)

Figure 5: Same as Figure 3, but for dependence of the mean transverse momentum $\left\langle p_{\mathrm{T}}\right\rangle$ on centrality $C$.

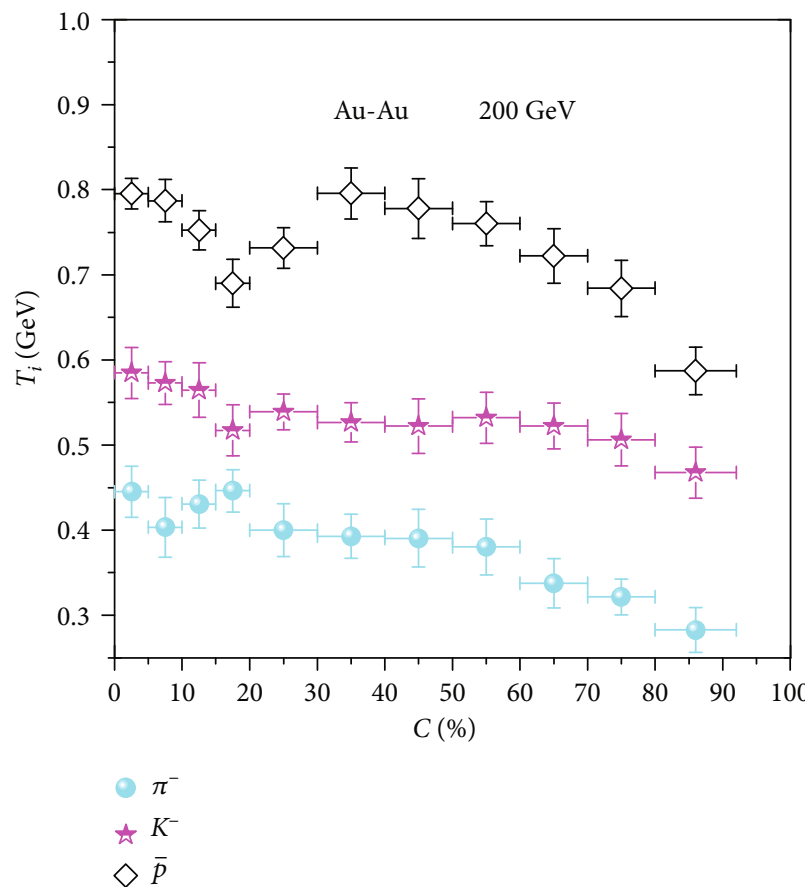

(a)

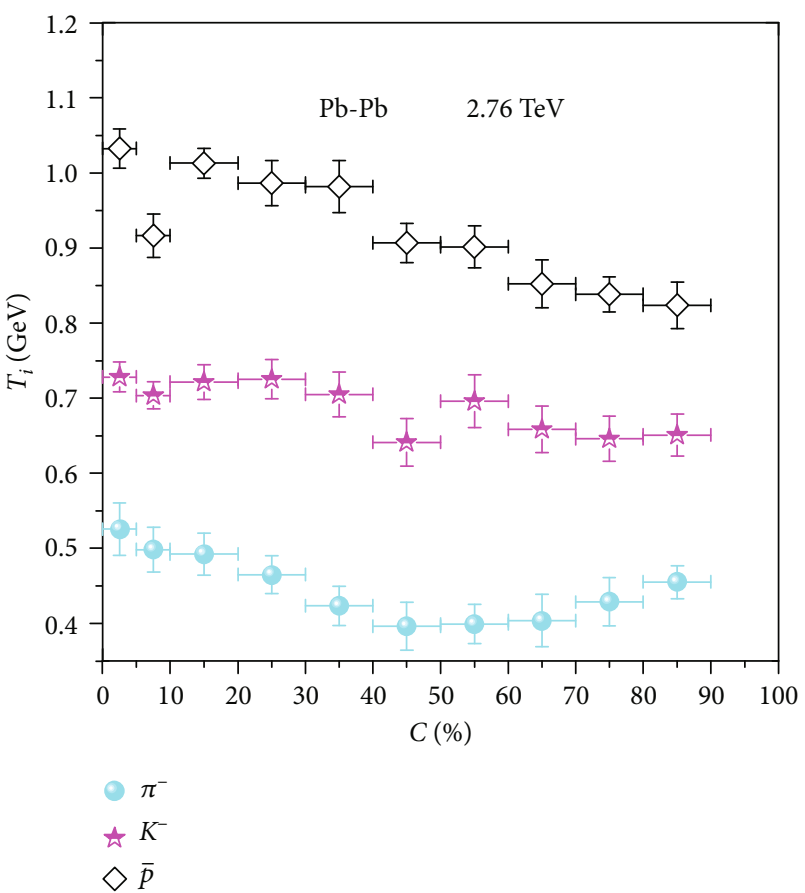

(b)

Figure 6: Same as Figure 3, but for dependence of the initial temperature $T_{i}$ on centrality $C$.

Indeed, the kinetic freeze-out temperature and transverse flow velocity and other quantities are model dependent. We notice that the current blast-wave model uses a small or almost zero transverse flow velocity in peripheral collisions and obtains a larger kinetic freeze-out tempera- ture in peripheral collisions compared to central collisions. If we use a large transverse flow velocity in peripheral collisions, we can obtain a smaller kinetic freeze-out temperature in peripheral collisions compared to central collisions [58]. 

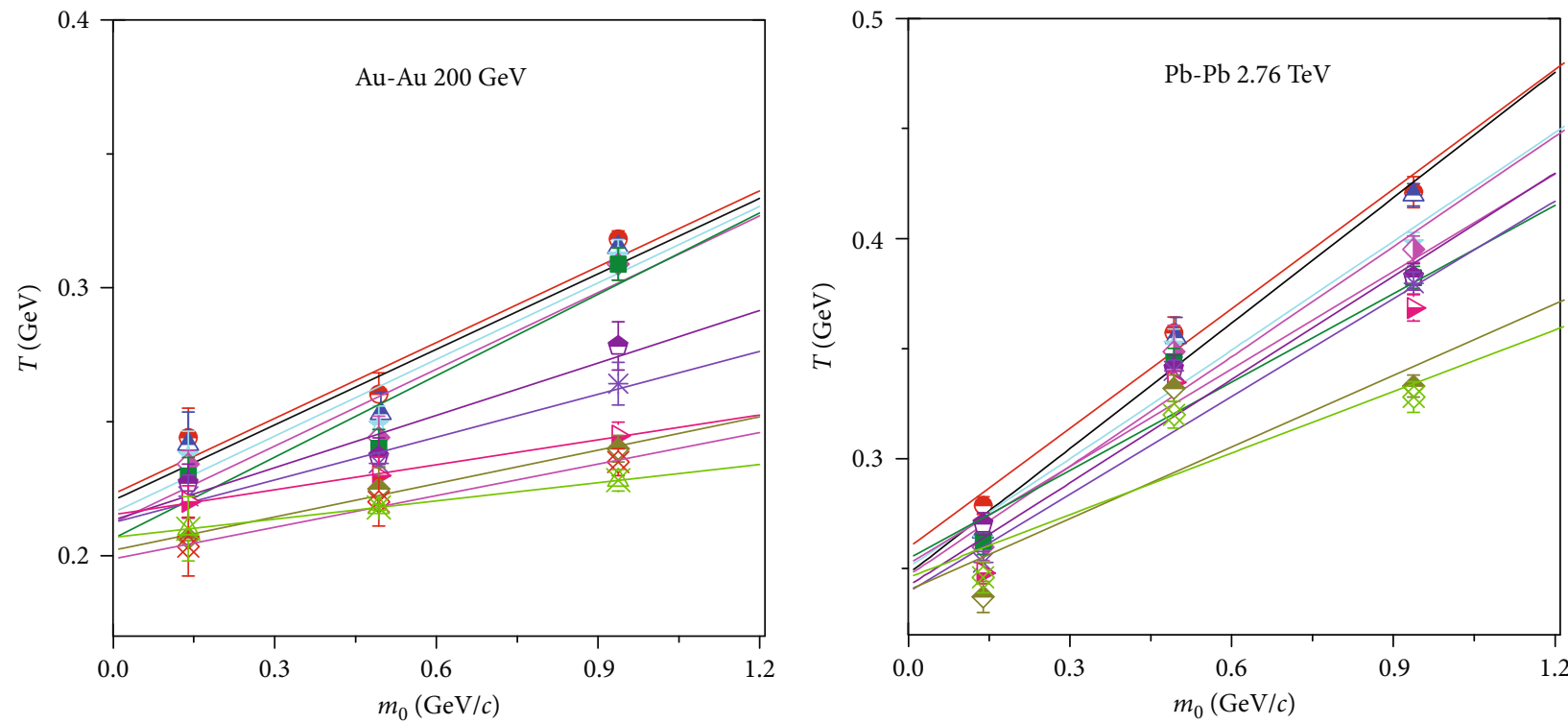

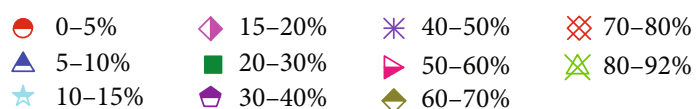

(a)

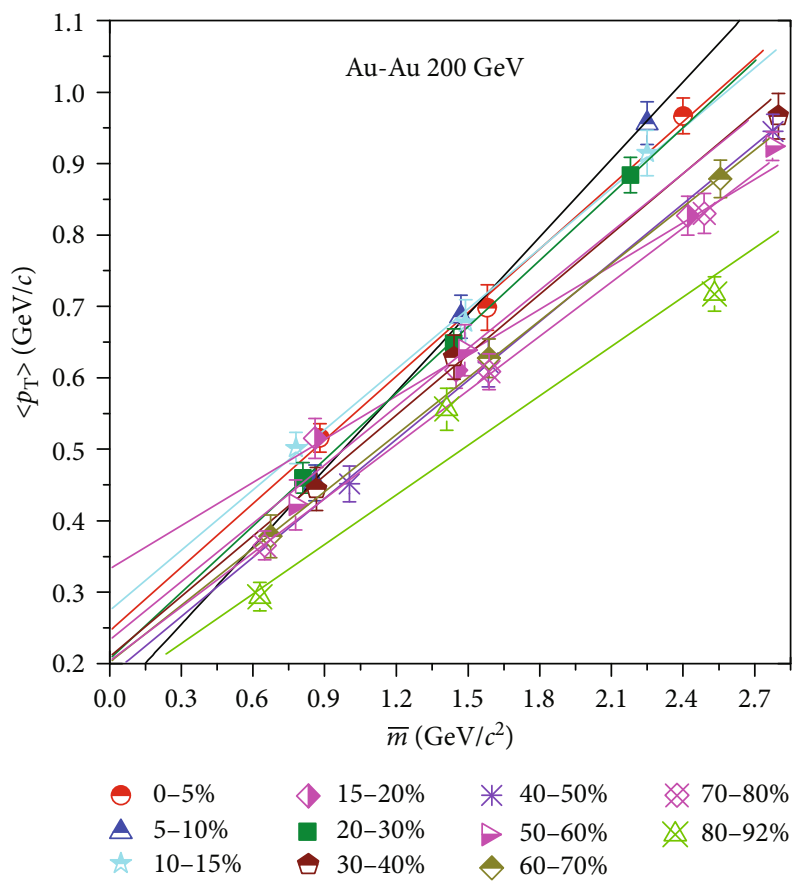

(c)

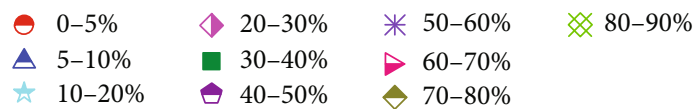

(b)

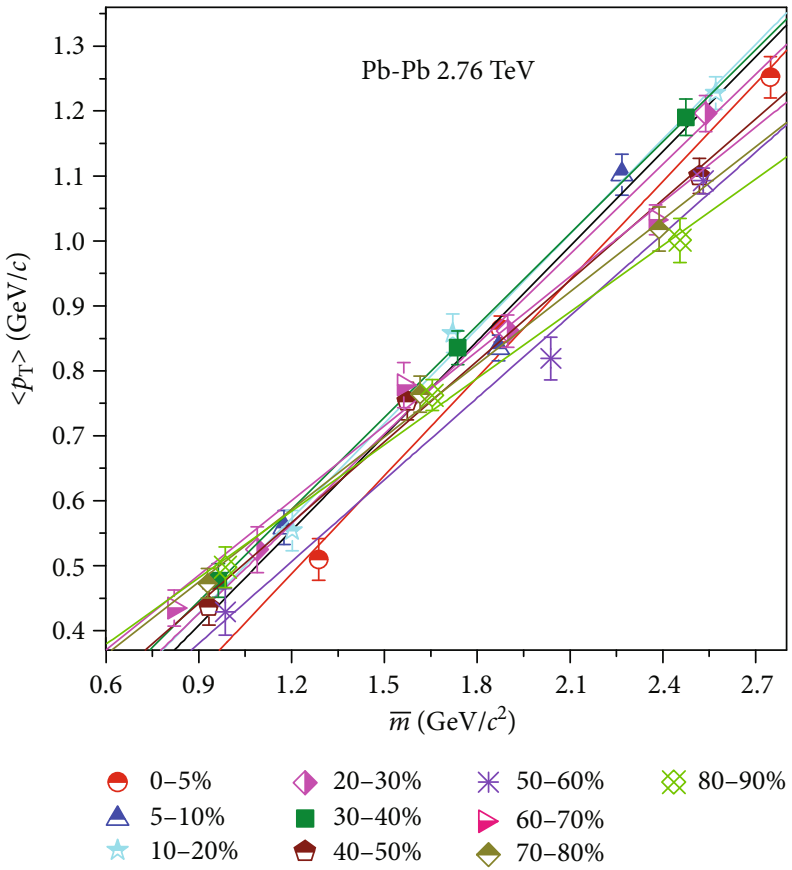

(d)

Figure 7: Dependence of $(\mathrm{a}, \mathrm{b})$ effective temperature $T$ on the rest mass $m_{0}$ and $(\mathrm{c}, \mathrm{d})$ mean transverse momentum $\left\langle p_{\mathrm{T}}\right\rangle$ on the mean energy or mean moving mass $\bar{m}$ for $(\mathrm{a}, \mathrm{c}) \mathrm{Au}-\mathrm{Au}$ collisions at $200 \mathrm{GeV}$ and $(\mathrm{b}, \mathrm{d}) \mathrm{Pb}-\mathrm{Pb}$ collisions at $2.76 \mathrm{TeV}$ in different centrality classes. The lines are the results fitted for the values of derived quantities.

In addition, the kinetic freeze-out temperature $T_{0}$ and transverse flow velocity $\beta_{\mathrm{T}}$ are also transverse momentum range dependent. In our opinion, to obtain the parameters as accurately as possible, we should use the transverse momentum range as accurately as possible. The transverse momentum range should not be too narrow or too wide. A too narrow transverse momentum range will exclude the contributions of some particles which should be included. A too wide transverse momentum range will include the contributions of some particles which should be excluded. In fact, model- and $p_{\mathrm{T}}$ range-independent $T_{0}$ and $\beta_{\mathrm{T}}$ are ideal. 


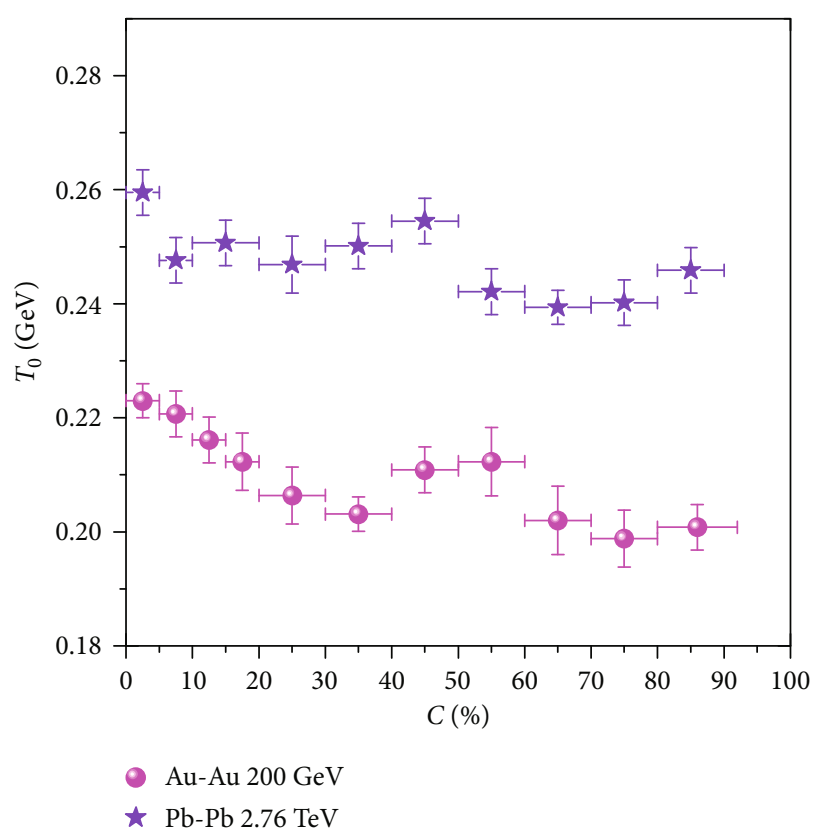

(a)

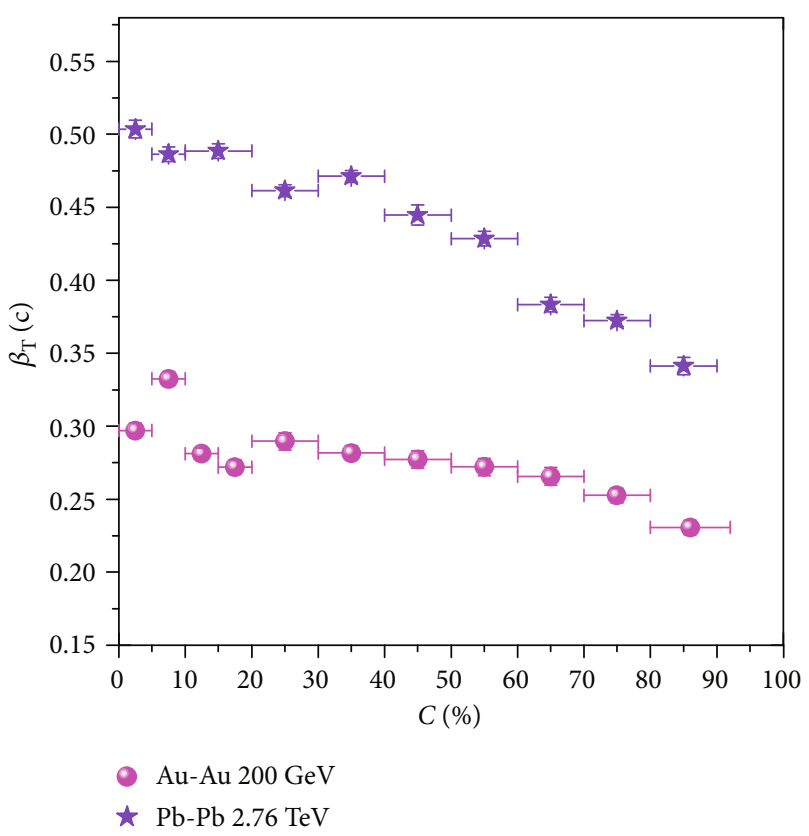

(b)

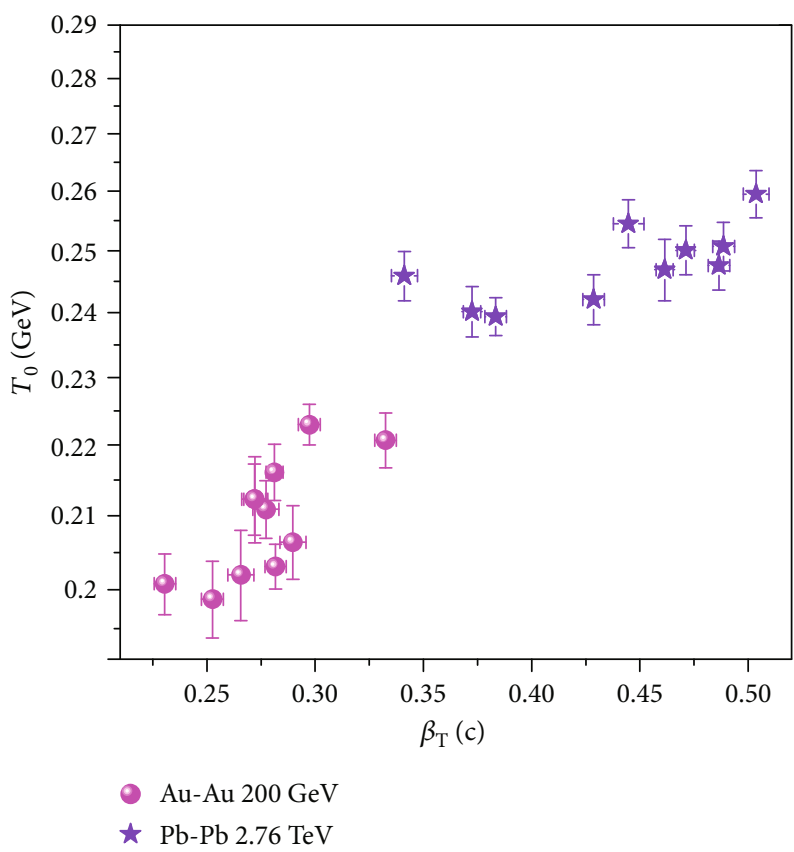

(c)

Figure 8: Dependence of (a) kinetic freeze-out temperature $T_{0}$ on centrality $C$ and (b) transverse flow velocity $\beta_{\mathrm{T}}$ on centrality $C$.

Generally, the mean transverse momentum $\left\langle p_{\mathrm{T}}\right\rangle$ and the root-mean-square transverse momentum $\sqrt{\left\langle p_{\mathrm{T}}^{2}\right\rangle}$ are model independent. Obtaining the initial temperature by $T_{i}=$ $\sqrt{\left\langle p_{\mathrm{T}}^{2}\right\rangle / 2}$ is a suitable treatment which is regardless of the model, though it is from the color string percolation model [15-17]. It is expected that $T_{0}$ and $\beta_{\mathrm{T}}$ are related to $\left\langle p_{\mathrm{T}}\right\rangle$, which results in model-independent $T_{0}$ and $\beta_{\mathrm{T}}$.

As what we did in our recent work [59], let $T_{0} \equiv k\left\langle p_{\mathrm{T}}\right\rangle / 2$ and $\beta_{\mathrm{T}} \equiv(1-k)\left\langle p_{\mathrm{T}}\right\rangle / 2 \bar{m}$, where $k$ is a parameter which can be approximately taken to be $0.3-0.01 \ln \left(\sqrt{s_{N N}}\right)\left(\sqrt{s_{N N}}\right.$ is in units of $\mathrm{GeV}$ ) [60], 1/2 is used due to both contributions of the projectile and target participants, and $\bar{m}$ denotes the mean energy of the considered particles in the source rest frame. If the $p_{\mathrm{T}}$ range is wide enough, $T_{0}$ and $\beta_{\mathrm{T}}$ are also $p_{\mathrm{T}}$ range independent.

If we use $T_{0}$ by the above new definition instead of the intercept in the linear relation between $T$ and $m_{0}$, the mean $T_{0}(\sim 0.10-0.12 \mathrm{GeV})$ obtained from Figure 9 is obviously less than that in the present work which is too large compared to others. Meanwhile, if we use $\beta_{\mathrm{T}}$ by the above new definition instead of the slope in the linear relation between $\left\langle p_{\mathrm{T}}\right\rangle$ and 


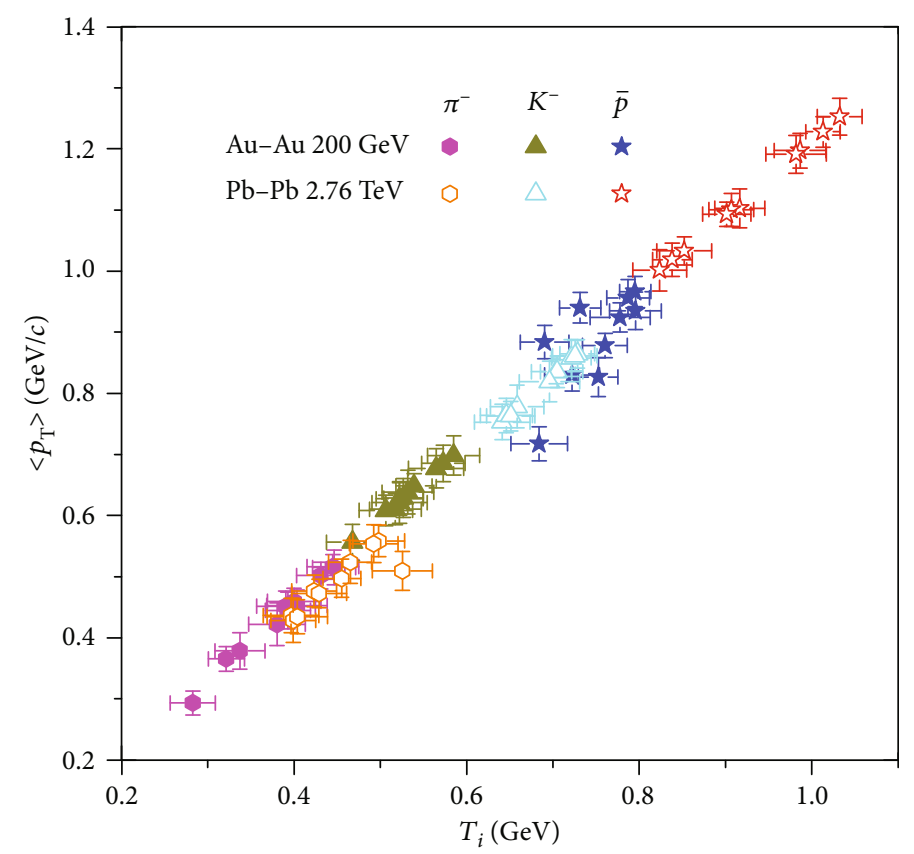

Figure 9: Dependence of the mean transverse momentum $\left\langle p_{\mathrm{T}}\right\rangle$ on the initial temperature $T_{i}$.

$\bar{m}$, the mean $\beta_{\mathrm{T}}(\sim 0.23-0.28 c)$ obtained from Figure 9 is less than that in the present work. Regardless of size, the new definitions of $T_{0}$ and $\beta_{\mathrm{T}}$ are model independent.

Before summary and conclusions, we would like to point out that this paper fits only the transverse momentum spectra measured from collisions with varying centralities by the two-component standard distribution. Some centrality dependences of related parameters are found. In our recent work [60-63], we have fitted the spectra measured from collisions with varying energies by the (twocomponent) standard distribution and/or Tsallis statistics. Some spectra are from small system size, and others are from large system size. The related parameters are found to depend also on energy and the larger nucleus in the projectile and target nuclei.

In particular, with the increasing energy, the kinetic freeze-out temperature increases quickly from a few $\mathrm{GeV}$ to around $10 \mathrm{GeV}$ and then slowly or slightly from around $10 \mathrm{GeV}$ to more than $10 \mathrm{TeV}$. This implies that around $10 \mathrm{GeV}$ is a special energy at which the interaction mechanism had changed. In fact, the collision system had undergone a baryon-dominated to meson-dominated final state [64]. This implies that the critical energy of phase transition from hadronic matter to QGP is possibly existent at around $10 \mathrm{GeV}$.

The dependence on the larger nucleus in the projectile and target nuclei is consistent to the dependence on centrality. This implies possibly that there is a critical size from the small to large systems and from the peripheral to central collisions. The data measured by the NA61/SHINE Collaboration [65] show that the nucleon number in the projectile or target nucleus on the onset of deconfinement is $\approx 10$. Meanwhile, the energy on the onset of deconfinement is $\approx 10 \mathrm{GeV}$. This double-10 signature is very interesting and should be studied further by various models and methods in the future.

We have studied three types of temperatures, namely, the effective temperature, initial temperature, and kinetic freezeout temperature, in this paper. Although the three types of temperatures are extracted from the transverse momentum spectra, they have different physics meanings. The effective temperature is obtained directly from the fit function, which describes together the degree of the thermal motion and flow effect at the stage of kinetic freeze-out. In the case of excluding the contribution of flow effect from the effective temperature, we expect to obtain the kinetic freeze-out temperature which describes only the thermal motion. The initial temperature in this paper is quoted directly from the color string percolation model [15-17], which is expected to describe the excitation degree of the initial state as what we did in our recent work [66].

It is regretful that the chemical freeze-out temperature is not discussed in this paper, though it has wider applications and discussions in the literature [67-69]. The chemical freeze-out temperature describes the excitation degree of the collision system at the stage of chemical freeze-out. Generally, the chemical freeze-out temperature can be obtained from the ratio of particle yields and can be used to map the phase diagram with the chemical potential. In the extensive statistics and/or axiomatic/generic nonextensive statistics [67-69], one may discuss the chemical and/or kinetic freeze-out parameters systematically.

\section{Conclusions}

The main observations and conclusions are summarized as follows: 
(a) The transverse momentum spectra of $\pi^{-}, K$, and $\bar{p}$ at mid(pseudo)rapidity produced in different centrality events in $\mathrm{Au}-\mathrm{Au}$ collisions at $200 \mathrm{GeV}$ and $\mathrm{Pb}-\mathrm{Pb}$ collisions at $2.76 \mathrm{TeV}$ have been analyzed. The experimental data measured by the PHENIX and ALICE Collaborations are fitted by the twocomponent standard distribution in which the temperature concept is quite close to the ideal gas model

(b) The effective temperature, initial temperature, kinetic freeze-out temperature, transverse flow velocity, and mean transverse momentum increase with the increase of event centrality from the peripheral to central collisions, which results in a higher excitation degree and quicker expansion velocity in central collisions. The kinetic freeze-out volume increases with the increase of event centrality from the peripheral to central collisions due to more number of participant nucleons taking part in central collisions

(c) The mass-dependent differential effective temperature, initial temperature, kinetic freeze-out volume, and mean transverse momentum are observed. The kinetic freeze-out temperature and transverse flow velocity extracted in this paper do not show a massdependent differential scenario due to the reason of methodology. Many quantities are model or method dependent

(d) The formation of similar systems is possible in the most peripheral nucleus-nucleus collisions at high energy and in the most central nucleus-nucleus collisions at low energy. This observation confirms the hypothesis of the effective energy for the particle production. There are many similarities in high-energy collisions

\section{Data Availability}

The data used to support the findings of this study are included within the article and are cited at relevant places within the text as references.

\section{Ethical Approval}

The authors declare that they are in compliance with ethical standards regarding the content of this paper.

\section{Disclosure}

The funding agencies have no role in the design of the study; in the collection, analysis, or interpretation of the data; in the writing of the manuscript; or in the decision to publish the results.

\section{Conflicts of Interest}

The authors declare that there are no conflicts of interest regarding the publication of this paper.

\section{Acknowledgments}

This work was supported by the National Natural Science Foundation of China under Grant Nos. 11575103 and 11947418, the Chinese Government Scholarship (China Scholarship Council), the Scientific and Technological Innovation Programs of Higher Education Institutions in Shanxi (STIP) under Grant No. 201802017, the Shanxi Provincial Natural Science Foundation under Grant No. 201901D111043, and the Fund for Shanxi "1331 Project" Key Subjects Construction.

\section{References}

[1] M. E. Fisher and A. N. Berker, "Scaling for first-order phase transitions in thermodynamic and finite systems," Physical Review B, vol. 26, no. 5, pp. 2507-2513, 1982.

[2] E. D. Davis and H. G. Miller, "“Phase transitions” at finite temperature in finite systems," Physics Letters B, vol. 196, no. 3, pp. 277-280, 1987.

[3] S. Mukherjee and V. Skokov, "Universality driven analytic structure of QCD crossover: radius of convergence in baryon chemical potential," https://arxiv.org/abs/1909.04639.

[4] W.-J. Fu, J. M. Pawlowski, and F. Rennecke, "The QCD phase structure at finite temperature and density," https://arxiv.org/ abs/1909.02991.

[5] G.-Y. Shao, W.-B. He, and X.-Y. Gao, "Deformed QCD phase structure and entropy oscillation in the presence of a magnetic background," Physical Review D, vol. 100, no. 1, article 014020, 2019.

[6] R. Sahoo, "Possible formation of QGP-droplets in protonproton collisions at the CERN Large Hadron Collider," AAPPS Bulletin, vol. 29, no. 4, pp. 16-21, 2019.

[7] M. Gyulassy and L. McLerran, "New forms of QCD matter discovered at RHIC," Nuclear Physics A, vol. 750, no. 1, pp. 30-63, 2005.

[8] M. T. AlFiky, O. ElSherif, and A. M. Hamed, "Onset of the jet quenching phenomenon," JETP Letters, vol. 111, no. 1, pp. 817, 2020.

[9] STAR Collaboration, "Bulk properties of the medium produced in relativistic heavy-ion collisions from the Beam Energy Scan program," Physical Review C, vol. 96, no. 4, article 044904, 2017.

[10] ALICE Collaboration, "Multiplicity dependence of pion, kaon, proton and lambda production in $\mathrm{p}-\mathrm{Pb}$ collisions at $\sqrt{s_{N N}}=$ 5.02 TeV," Physics Letters B, vol. 728, pp. 25-38, 2014.

[11] ALICE Collaboration, "Anisotropic flow in Xe-Xe collisions at $\sqrt{s_{N N}}=5.44$ TeV," Physics Letters B, vol. 784, pp. 82-95, 2018.

[12] L. Van Hove, "Two problems concerning hot hadronic matter and high energy collisions (Equilibrium formation, plasma deflagration)," Zeitschrift für Physik C Particles and Fields, vol. 21, no. 1, pp. 93-98, 1983.

[13] S. Chatterjee, S. Das, L. Kumar et al., "Freeze-out parameters in heavy-ion collisions at AGS, SPS, RHIC, and LHC energies," Advances in High Energy Physics, vol. 2015, 20 pages, 2015.

[14] S. Uddin, I. Bashir, and R. A. Bhat, "Transverse momentum distributions of hadrons produced in $\mathrm{Pb}-\mathrm{Pb}$ collisions at LHC energy $\sqrt{s_{N N}}=2.76 \mathrm{TeV}$," Advances in High Energy Physics, vol. 2015, Article ID 154853, 7 pages, 2015.

[15] L. J. Gutay, A. S. Hirsch, R. P. Scharenberg, B. K. Srivastava, and C. Pajares, "De-confinement in small systems: Clustering 
of color sources in high multiplicity $\bar{p} p$ collisions at $\sqrt{s}=1.8$ TeV," International Journal of Modern Physics E, vol. 24, no. 12, article 1550101, 2015.

[16] A. S. Hirsch, C. Pajares, R. P. Scharenberg, and B. K. Srivastava, "Exploring the initial stage of high multiplicity proton-proton collisions by determining the initial temperature of the quark-gluon plasma," Physical Review D, vol. 100, no. 11, article 114040, 2019.

[17] P. Sahoo, S. De, S. K. Tiwari, and R. Sahoo, "Energy and centrality dependent study of deconfinement phase transition in a color string percolation approach at RHIC energies," The European Physical Journal A, vol. 54, no. 8, p. 136, 2018.

[18] D. H. E. Gross, "Microcanonical thermodynamics and statistical fragmentation of dissipative systems. The topological structure of the N-body phase space," Physics Reports, vol. 279, no. 3-4, pp. 119-201, 1997.

[19] B. Borderie, "Dynamics and thermodynamics of the liquid-gas phase transition in hot nuclei studied with the INDRA array," Journal of Physics G: Nuclear and Particle Physics, vol. 28, no. 8, pp. R217-R247, 2002.

[20] M. D’Agostino, F. Gulminelli, P. Chomaz et al., "Negative heat capacity in the critical region of nuclear fragmentation: an experimental evidence of the liquid-gas phase transition," Physics Letters B, vol. 473, no. 3-4, pp. 219-225, 2000.

[21] M. D'Agostino, R. Bougault, F. Gulminelli et al., "On the reliability of negative heat capacity measurements," Nuclear Physics $A$, vol. 699, no. 3-4, pp. 795-818, 2002.

[22] P. Chomaz, V. Duflot, and F. Gulminelli, "Caloric curves and energy fluctuations in the microcanonical liquid-gas phase transition," Physical Review Letters, vol. 85, no. 17, pp. 35873590, 2000.

[23] R. Hagedorn, "Multiplicities, $p_{T}$ distributions and the expected hadron $\rightarrow$ quark-gluon phase transition," La Rivista del Nuovo Cimento, vol. 6, no. 10, pp. 1-50, 1983.

[24] J. Cleymans and D. Worku, "Relativistic thermodynamics: transverse momentum distributions in highenergy physics," The European Physical Journal A, vol. 48, no. 11, p. 160, 2012.

[25] E. Schnedermann, J. Sollfrank, and U. Heinz, "Thermal phenomenology of hadrons from $200 \mathrm{~A} \mathrm{GeV} \mathrm{S+S} \mathrm{collisions,"} \mathrm{Phys-}$ ical Review C, vol. 48, no. 5, pp. 2462-2475, 1993.

[26] STAR Collaboration, "Systematic measurements of identified particle spectra in $p p, d+\mathrm{Au}$ and $\mathrm{Au}+\mathrm{Au}$ collisions from STAR,” Physical Review C, vol. 79, article 034909, 2009.

[27] STAR Collaboration, "Identified particle production, azimuthal anisotropy, and interferometry measurements in $\mathrm{Au}+\mathrm{Au}$ collisions at $\sqrt{s_{N N}}=9.2 \mathrm{GeV}$," Physical Review C, vol. 81, article 024911, 2010.

[28] Z. Tang, Y. Xu, L. Ruan, G. van Buren, F. Wang, and Z. Xu, "Spectra and radial flow in relativistic heavy ion collisions with Tsallis statistics in a blast-wave description," Physical Review C, vol. 79, no. 5, article 051901(R), 2009.

[29] Z.-B. Tang, L. Yi, L. J. Ruan et al., "The Statistical origin of constituent-quark scaling in QGP hadronization," Chinese Physics Letters, vol. 30, no. 3, article 031201, 2013.

[30] K. Jiang, Y. Zhu, W. Liu et al., "Onset of radial flow in $p$ +pcollisions," Physical Review C, vol. 91, no. 2, article 024910, 2015.

[31] J. Cleymans, H. Oeschler, K. Redlich, and S. Wheaton, "Comparison of chemical freeze-out criteria in heavyion collisions," Physical Review C, vol. 73, no. 3, article 034905, 2006.
[32] A. Andronic, P. Braun-Munzinger, and J. Stachel, "Hadron production in central nucleus-nucleus collisions at chemical freeze-out," Nuclear Physics A, vol. 772, no. 3-4, pp. 167-199, 2006.

[33] S. Uddin, J. S. Ahmad, W. Bashir, and R. A. Bhat, “A unified approach towards describing rapidity and transverse momentum distributions in a thermal freeze-out model," Journal of Physics G: Nuclear and Particle Physics, vol. 39, no. 1, article 015012, 2012.

[34] R. P. Adak, S. Das, S. K. Ghosh, R. Ray, and S. Samanta, "Centrality dependence of chemical freeze out parameters from netproton and net-charge fluctuations using a hadron resonance gas model," Physical Review C, vol. 96, no. 1, article 014902, 2017.

[35] R. Odorico, "Does a transverse energy trigger actually trigger on large- $P_{\mathrm{T}}$ jets?," Physics Letters $B$, vol. 118, no. 1-3, pp. 151-154, 1982.

[36] ALICE Collaboration, "Transverse momentum spectra of charged particles in proton-proton collisions at $\sqrt{s}=900 \mathrm{GeV}$ with ALICE at the LHC," Physics Letters B, vol. 693, pp. 53-68, 2010.

[37] M. Biyajima, T. Mizoguchi, and N. Suzuki, "Analyses of whole transverse momentum distributions in $p \bar{p}$ and $p p$ collisions by using a modified version of Hagedorn's formula," International Journal of Modern Physics A, vol. 32, article 1750057, 2017.

[38] PHENIX Collaboration, "Identified charged particle spectra and yields in $\mathrm{Au}+\mathrm{Au}$ collisions at $\sqrt{s_{N N}}=200 \mathrm{GeV}$," Physical Review C, vol. 69, article 034909, 2004.

[39] ALICE Collaboration, "Centrality dependence of $\pi, K$, and $p$ production in $\mathrm{Pb}-\mathrm{Pb}$ collisions at $\sqrt{s_{N N}}=2.76 \mathrm{TeV}$," Physical Review C, vol. 88, article 044910, 2013.

[40] D. Thakur, S. Tripathy, P. Garg, R. Sahoo, and J. Cleymans, "Indication of differential kinetic freeze-out at RHIC and LHC energies," Acta Physica Polonica B Proceedings Supplement, vol. 9, no. 2, pp. 329-332, 2016.

[41] D. Thakur, S. Tripathy, P. Garg, R. Sahoo, and J. Cleymans, "Indication of a differential freeze-out in proton-proton and heavy-ion collisions at RHIC and LHC energies," Advances in High Energy Physics, vol. 2016, Article ID 4149352, 13 pages, 2016.

[42] PHENIX Collaboration, "Centrality dependence of $\pi^{+/-}$, $K^{+/-}, p$ and $\bar{p}$ production from $\sqrt{s_{N N}}=13 \mathrm{GeV} \mathrm{Au}+\mathrm{Au}$ collisions at RHIC," Physical Review Letters, vol. 88, article 242301, 2002.

[43] UA1 Collaboration, "A study of the genera characteristics of $\bar{p}$ collisions at $s=0.2$ to $0.9 \mathrm{TeV}$," Nuclear Physics B, vol. 335, pp. 261-287, 1990.

[44] E802 Collaboration, "Particle production in Au+Au collisions from BNL E866," Nuclear Physics A, vol. 610, pp. 139-152, 1996.

[45] NA44 Collaboration, "Collective expansion in high energy heavy ion collisions," Physical Review Letters, vol. 78, pp. 2080-2083, 1997.

[46] P.-P. Yang, Q. Wang, and F.-H. Liu, "Mutual derivation between arbitrary distribution forms of momenta and momentum components," International Journal of Theoretical Physics, vol. 58, no. 8, pp. 2603-2618, 2019.

[47] STAR Collaboration, "Systematic measurements of identified particle spectra in $p p, d+\mathrm{Au}$, and $\mathrm{Au}+\mathrm{Au}$ collisions at the STAR detector," Physical Review C, vol. 79, article 034909, 2009. 
[48] S. Takeuchi, K. Murase, T. Hirano, P. Huovinen, and Y. Nara, "Effects of hadronic rescattering on multistrange hadrons in high-energy nuclear collisions," Physical Review C, vol. 92, no. 4, article 044907, 2015.

[49] H.-R. Wei, F.-H. Liu, and R. A. Lacey, "Disentangling random thermal motion of particles and collective expansion of source from transverse momentum spectra in high energy collisions," Journal of Physics G: Nuclear and Particle Physics, vol. 43, no. 12, article 125102, 2016.

[50] H.-R. Wei, F.-H. Liu, and R. A. Lacey, "Kinetic freeze-out temperature and flow velocity extracted from transverse momentum spectra of final-state light flavor particles produced in collisions at RHIC and LHC," The European Physical Journal $A$, vol. 52, no. 4, p. 102, 2016.

[51] E. K. G. Sarkisyan and A. S. Sakharov, "Relating multihadron production in hadronic and nuclear collisions," The European Physical Journal C, vol. 70, no. 3, pp. 533-541, 2010.

[52] A. N. Mishra, R. Sahoo, E. K. G. Sarkisyan, and A. S. Sakharov, "Effective-energy budget in multiparticle production in nuclear collisions," The European Physical Journal C, vol. 74, no. 11, article 3147, 2014and "Erratum," The European Physical Journal C, vol. 75, article 70, 2015.

[53] E. K. Sarkisyan-Grinbaum, A. Nath Mishra, R. Sahoo, and A. S. Sakharov, "Effective-energy universality approach describing total multiplicity centrality dependence in heavyion collisions," EPL, vol. 127, no. 6, article 62001, 2019.

[54] E. K. G. Sarkisyan, A. N. Mishra, R. Sahoo, and A. S. Sakharov, "Multihadron production dynamics exploring the energy balance in hadronic and nuclear collisions," Physical Review D, vol. 93, no. 5, article 054046, 2016and "Addendum," Physical Review D, vol. 93, Article ID 079904, 2016.

[55] D. Prorok, "Centrality dependence of freeze-out temperature fluctuations in $\mathrm{Pb}-\mathrm{Pb}$ collisions at the LHC," The European Physical Journal A, vol. 55, no. 3, p. 37, 2019.

[56] B. De, "Non-extensive statistics and understanding particle production and kinetic freeze-out process from $p_{T-}$ spectra at 2.76 TeV," The European Physical Journal A, vol. 50, no. 9, article 138, 2014.

[57] R. Pasechnik and M. Sumbera, "Phenomenological review on quark-gluon plasma: concepts vs. observations," Universe, vol. 3, no. 1, p. 7, 2017.

[58] H.-L. Lao, F.-H. Liu, B.-C. Li, and M.-Y. Duan, "Kinetic freezeout temperatures in central and peripheral collisions: which one is larger?," Nuclear Science and Techniques, vol. 29, no. 6 , p. 82,2018 .

[59] M. Waqas and F.-H. Liu, "Initial, effective, and kinetic freeze-out temperatures from transverse momentum spectra in high-energy proton(deuteron)-nucleus and nucleusnucleus collisions," The European Physical Journal Plus, vol. 135, no. 2, p. 147, 2020.

[60] L.-L. Li, F.-H. Liu, M. Waqas, R. Al-Yusufi, and A. Mujear, "Excitation functions of related parameters from transverse momentum (mass) spectra in high energy collisions," 2019, http://arxiv.org/abs/1911.07419.

[61] L.-L. Li and F.-H. Liu, "Energy dependent kinetic freeze-out temperature and transverse flow velocity in high energy collisions," The European Physical Journal A, vol. 54, no. 10, p. 169, 2018.

[62] L.-L. Li and F.-H. Liu, "Excitation functions of kinetic freezeout temperature and transverse flow velocity in protonproton collisions," http://arxiv.org/abs/1805.03342.
[63] H.-L. Lao, F.-H. Liu, B.-C. Li, M.-Y. Duan, and R. A. Lacey, "Examining the model dependence of the determination of kinetic freeze-out temperature and transverse flow velocity in small collision system," Nuclear Science and Techniques, vol. 29, no. 11, p. 164, 2018.

[64] J. Cleymans, "The physics case for the $s_{N N} \approx 10 \mathrm{GeV}$ energy region," in Walter Greiner Memorial Volume, P. O. Hess, Ed., World Scientiflc, Singapore, 2018.

[65] M. P. Lewicki and L. Turko, "NA61/SHINE shining more light on the onset of deconfinement," CERN EP Newsletter, pp. 6772, 2019, http://arxiv.org/abs/2002.00631.

[66] Q. Wang and F.-H. Liu, "Initial and final state temperatures of antiproton emission sources in high energy collisions," International Journal of Theoretical Physics, vol. 58, no. 12, pp. 4119-4138, 2019.

[67] A. N. Tawfik, H. Yassin, and E. R. Abo Elyazeed, "Extensive/nonextensive statistics for $p_{T}$ distributions of various charged particles produced in $\mathrm{p}+\mathrm{p}$ and $\mathrm{A}+\mathrm{A}$ collisions in a wide range of energies," http://arxiv.org/abs/1905.12756.

[68] A. N. Tawfik, "Axiomatic nonextensive statistics at NICA energies," The European Physical Journal A, vol. 52, no. 8, p. 253, 2016.

[69] A. Tawfik, H. Yassin, and E. R. Abo Elyazeed, "Chemical freezeout parameters within generic nonextensive statistics," Indian Journal of Physics, vol. 92, no. 10, pp. 1325-1335, 2018. 